\title{
The Gaia-ESO Survey: Lithium enrichment histories of the Galactic thick and thin disc ${ }^{\star}$
}

\author{
X. Fu ${ }^{1,2}$, D. Romano ${ }^{2}$, A. Bragaglia ${ }^{2}$, A. Mucciarelli ${ }^{1,2}$, K. Lind ${ }^{3}$, E. Delgado Mena ${ }^{4}$, S. G. Sousa ${ }^{4}$, S. Randich ${ }^{5}$, \\ A. Bressan ${ }^{6}$, L. Sbordone ${ }^{7}$, S. Martell ${ }^{8}$, A. J. Korn ${ }^{9}$, C. Abia ${ }^{10}$, R. Smiljanic ${ }^{11}$, P. Jofré ${ }^{12,25}$, E. Pancino ${ }^{5}$, \\ G. Tautvaišiené $\dot{e}^{13}$, B. Tang ${ }^{14}$, L. Magrini ${ }^{5}$, A. C. Lanzafame $^{15}$, G. Carraro ${ }^{16}$, T. Bensby ${ }^{17}$, F. Damiani ${ }^{18}$, E. J. Alfaro ${ }^{19}$, \\ E. Flaccomio ${ }^{18}$, L. Morbidelli ${ }^{5}$, S. Zaggia ${ }^{20}$, C. Lardo $^{21}$, L. Monaco $^{22}$, A. Frasca ${ }^{15}$, P. Donati ${ }^{2}$, A. Drazdauskas ${ }^{13}$, \\ Y. Chorniy ${ }^{13}$, A. Bayo ${ }^{23}$, and G. Kordopatis ${ }^{24}$ \\ (Affiliations can be found after the references)
}

Received 30 July 2017 / Accepted 9 November 2017

\begin{abstract}
Lithium abundance in most of the warm metal-poor main sequence stars shows a constarnt plateau $(A(\mathrm{Li}) \sim 2.2$ dex $)$ and then the upper envelope of the lithium vs. metallicity distribution increases as we approach solar metallicity. Meteorites, which carry information about the chemical composition of the interstellar medium (ISM) at the solar system formation time, show a lithium abundance $A(\mathrm{Li}) \sim 3.26$ dex. This pattern reflects the Li enrichment history of the ISM during the Galaxy lifetime. After the initial Li production in big bang nucleosynthesis, the sources of the enrichment include asymptotic giant branch (AGB) stars, low-mass red giants, novae, type II supernovae, and Galactic cosmic rays. The total amount of enriched $\mathrm{Li}$ is sensitive to the relative contribution of these sources. Thus different $\mathrm{Li}$ enrichment histories are expected in the Galactic thick and thin disc. We investigate the main sequence stars observed with UVES in Gaia-ESO Survey iDR4 catalogue and find a Li$[\alpha / \mathrm{Fe}]$ anticorrelation independent of $[\mathrm{Fe} / \mathrm{H}], T_{\text {eff }}$, and $\log (g)$. Since in stellar evolution different $\alpha$ enhancements at the same metallicity do not lead to a measurable $\mathrm{Li}$ abundance change, the anticorrelation indicates that more $\mathrm{Li}$ is produced during the Galactic thin disc phase than during the Galactic thick disc phase. We also find a correlation between the abundance of Li and s-process elements Ba and Y, and they both decrease above the solar metallicity, which can be explained in the framework of the adopted Galactic chemical evolution models.
\end{abstract}

Key words. stars: abundances - Galaxy: abundances - Galaxy: disk

\section{Introduction}

The evolution of lithium $\left({ }^{7} \mathrm{Li}\right)$ in the Galaxy is a critical and not well understood issue, because of the many unknowns that still affect the proposed production and destruction channels of this element. Most of the metal-poor $(-2.4 \lesssim[\mathrm{Fe} / \mathrm{H}] \lesssim-1.4)$, warm $\left(5700 \mathrm{~K} \lesssim T_{\text {eff }} \lesssim 6800 \mathrm{~K}\right)$ Galactic halo dwarfs are known to share a very similar ${ }^{7} \mathrm{Li}$ abundance; the so-called "Spite plateau" $(A(\mathrm{Li}))^{1} \simeq 2.05-2.2$ dex, Spite \& Spite 1982; Bonifacio \& Molaro 1997; Asplund et al. 2006; Bonifacio et al. 2007), which for a long time has been thought to represent the primordial $\mathrm{Li}$ abundance produced in the big bang nucleosynthesis. However, standard big bang nucleosynthesis (SBBN) predicts that the primordial $\mathrm{Li}$ abundance is mainly determined by the primordial baryon-to-photon ratio, which can be derived from the acoustic oscillations of the cosmic microwave background (CMB), and the baryon-to-photon ratio obtained by the Planck satellite data leads to $A(\mathrm{Li})=2.66-2.73 \mathrm{dex}$ (Coc et al. 2014), that is, the SBBN predicted primordial Li abundance exceeds the stellar observationally inferred value by a factor of almost three. Furthermore, the Spite plateau is not constant, but bends down for extremely metal-poor stars $([\mathrm{Fe} / \mathrm{H}] \lesssim-2.8$ dex, e.g. Sbordone et al. 2010; Melendez et al. 2010; Hansen et al. 2014; Bonifacio et al. 2015). The environmental ${ }^{7} \mathrm{Li}$ evolution

\footnotetext{
* The full Table 1 is only available at the CDS via anonymous ftp to cdsarc.u-strasbg. fr (130.79.128.5) or via

http://cdsarc.u-strasbg.fr/viz-bin/qcat?]/A+A/610/A38

$1 \quad A(\mathrm{Li})=12+\log [n(\mathrm{Li}) / n(\mathrm{H})]$ where $n$ is the number density of atoms and 12 is the solar hydrogen abundance.
}

model proposed by Fu et al. (2015) offers a way to reconcile the SBBN theory to the observations of stars on the plateau by considering the pre-main sequence accretion, overshooting, and main sequence diffusion, while also accounting for the drop of ${ }^{7} \mathrm{Li}$ abundances in extremely metal-poor stars, but it needs to be further studied and better constrained (for instance, by including the effects of stellar rotation).

As far as the Galactic disc stars are concerned, a large dispersion is seen in the data (see Ramírez et al. 2012; Delgado Mena et al. 2015; Guiglion et al. 2016, for recent works). The observed scatter is due to efficient ${ }^{7} \mathrm{Li}$ destruction in stellar interiors (e.g. $A_{\mathrm{Li}}=1.05 \mathrm{dex}$ in the Sun, Grevesse et al. 2007), coupled to non-negligible ${ }^{7} \mathrm{Li}$ production on a Galactic scale. The discovery of unevolved stars with high ${ }^{7} \mathrm{Li}$ abundances (comparable to or higher than those observed in meteorites, $A(\mathrm{Li})=3.26$ dex, Lodders et al. 2009) dates back to long ago (see e.g. Bonsack \& Greenstein 1960; Herbig 1965; Wallerstein et al. 1965, for T Tauri, F and G dwarf, and Hyades main-sequence stars, respectively), and it is now commonly interpreted as a signature of ${ }^{7} \mathrm{Li}$ enrichment of the interstellar medium (ISM), due to various production processes. Indeed, ${ }^{7} \mathrm{Li}$ is synthesised in different astrophysical sites, apart from the big bang:

- High-energy processes involving Galactic cosmic rays (GCRs) hitting the ISM atoms contribute less than 20$30 \%$ of the meteoritic ${ }^{7} \mathrm{Li}$ abundance (Reeves et al. 1970; Meneguzzi et al. 1971; Lemoine et al. 1998; Romano et al. 2001; Prantzos 2012), meaning that about $70 \%$ of the Solar 
System ${ }^{7} \mathrm{Li}$ must originate from thermonuclear reactions acting in the stellar interiors.

- As first suggested by Domogatskii et al. (1978), the $v$ process may lead to the synthesis of ${ }^{7} \mathrm{Li}$ in the helium shell of core-collapse supernovae (SNe). However, this mechanism ought to work quite inefficiently at a Galactic level (see discussions in Vangioni-Flam et al. 1996; Romano et al. 1999); furthermore, to the best of our knowledge there is no observational evidence in support of it.

- Observations in both the Milky Way (disc, bulge, halo, Globular Clusters; e.g. Wallerstein \& Sneden 1982; Pilachowski 1986; Hill \& Pasquini 1999; Kraft et al. 1999; Balachandran et al. 2000; Kumar \& Reddy 2009; Monaco et al. 2011; D'Orazi et al. 2015; Casey et al. 2016; Kirby et al. 2016) and Local Group dwarf galaxies (Domínguez et al. 2004; Kirby et al. 2012) have conclusively shown that a number of Li-rich giants do exist with abundances higher than the standard stellar evolution theory predicts $(A(\mathrm{Li}) \sim 1.0 \mathrm{dex}$ at the beginning of the Red Giant Branch bump for Pop. II stars around $1 M_{\odot}$ (Charbonnel \& Zahn 2007; Fu 2016), and even lower for more massive stars Iben, Icko 1967a,b), or even exceeding the SBBN prediction and the meteorites' value, indicating that the Li in these stars must have been created rather than preserved from destruction. Stars can produce ${ }^{7} \mathrm{Li}$ in their late stages of evolution via the Cameron-Fowler mechanism (Cameron \& Fowler 1971), which may work both in intermediate-mass stars on the asymptotic giant branch (AGB; Sackmann \& Boothroyd 1992) and in low-mass stars on the red giant branch (RGB), under special conditions (Sackmann \& Boothroyd 1999). Abia et al. (1993) conclude that the contribution of carbon stars to the Galactic $\mathrm{Li}$ enrichment can reach $30 \%$ depending on the mass loss and $\mathrm{Li}$ production in the Li-rich AGB stars. However, the detailed mechanism(s) of the Li enrichment in these giant stars remains unknown, and we lack a clear and unequivocal assessment of the relative contributions of these two stellar contributions to the overall Galactic lithium enrichment: For example, Romano et al. (2001) and Travaglio et al. (2001) reach opposite conclusions, because of the adoption of different yield sets and different assumptions about the underlying stellar physics (e.g. mixing process, mass-loss rate) in their chemical evolution models.

- Another, potentially major source of ${ }^{7} \mathrm{Li}$ is nova systems, which are able to produce it when a thermonuclear runaway occurs in the hydrogen envelope of the accreting white dwarf, as proposed long ago by Starrfield et al. (1978). Notwithstanding the considerable observational efforts, a direct detection of ${ }^{7} \mathrm{Li}$ during a nova outburst remained elusive until the very recent detection of the blueshifted Li I $\lambda 6708 \AA$ line in the spectrum of the nova V1369 Cen by Izzo et al. (2015). Based on the intensity of the absorption line and on current estimates of the Galactic nova rate, Izzo et al. (2015) estimate that novae might be able to explain most of the enriched lithium observed in the young stellar populations. Further support in favour of a high production of ${ }^{7} \mathrm{Li}$ during nova outbursts comes from the detection of highly enriched ${ }^{7} \mathrm{Be}$ (later decaying to ${ }^{7} \mathrm{Li}$ ) in the ejecta of novae V339 Del, V2944 Oph, and V5668 Sgr by Tajitsu et al. (2015) and Tajitsu et al. (2016), respectively (see also Molaro et al. 2016).

In this paper, we investigate the lithium content and enrichment history in (mostly) thin and thick disc stars in the Milky Way, as well as its relationships with the abundances of selected $\alpha$ and $s$-process elements. To do so, we take advantage of UVES spectra analysed by the Gaia-ESO consortium for 1399 main sequence stars. Furthermore, we use an updated version of the model presented by Romano et al. (1999, 2001), which takes all of the above-mentioned sources of lithium into account (see also Izzo et al. 2015), to discuss our ${ }^{7} \mathrm{Li}$ measurements. In particular, we show that, in principle, in the framework of such a model it is possible to offer an explanation for the observed peculiar trend of decreasing ${ }^{7} \mathrm{Li}$ for super-solar metallicity stars (Delgado Mena et al. 2015; Guiglion et al. 2016), if it is indeed real.

The paper is organised as follows. In Sect. 2 we describe the observations, data analysis, and sample selection. The main results are presented in Sect. 3, including the trends of $\mathrm{Li}$ abundances in thin and thick disc stars, also in relation with the $\alpha$ and s-process elements behaviours. In Sect. 4 we discuss the Galactic chemical evolution of Li. The conclusions and a final summary are given in Sect. 5 .

\section{Gaia-ESO Survey observations and data analysis}

The Gaia-ESO Survey (GES hereafter, Gilmore et al. 2012; Randich et al. 2013) is a large, public, high-resolution spectroscopic survey using the FLAMES facility at ESO Very Large Telescope (VLT); that is, it simultaneously employs the UVES and GIRAFFE spectrographs (Dekker et al. 2000; Pasquini et al. 2000). GES is intended to complement the Gaia astrometric and photometric data of exquisite precision (Gaia Collaboration et al. 2016) with radial velocities and detailed chemistry for about $10^{5}$ stars of all major Galactic populations and about 70 open clusters (which will not be considered further in this paper since the purpose here is to investigate the chemical evolution of the field stars).

To study the Milky Way (MW) field population, GES uses mostly the medium-resolution spectrograph GIRAFFE with two setups (HR10, HR21). However, GES also observes MW field stars with UVES and a setup centred at 580nm $(\lambda=480$ $680 \mathrm{~nm}, R \sim 47000)$ and is collecting high-resolution, high signal-to-noise $(\mathrm{S} / \mathrm{N})$ spectra of a few thousand FG-type stars in the solar neighbourhood (within about $2 \mathrm{kpc}$ from the Sun). This sample includes mainly disc stars of all ages and metallicities, plus a small fraction of local halo stars, and is intended to produce a detailed chemical and kinematical characterization to fully complement the results of the Gaia mission, which achieve their maximum precision within this distance limit.

The detailed description of the MW sample selection is presented in Stonkute et al. (2016). In brief, the UVES observations are made in parallel with the GIRAFFE ones and their position and exposure time are dictated by the latter. The 2MASS infrared photometry (Skrutskie et al. 2006) was used to select dwarfs and main sequence turn-off stars of FG spectral type, with a limiting magnitude of $J_{2 \mathrm{MASS}}=14$ and a narrow colour range. The selection takes into account also the effects of interstellar extinction, estimated through the reddening maps of Schlegel et al. (1998). Though some remaining uncertainties still affect the evolutionary phases of the stars, the majority of the observed targets turn out to be dwarf stars, as we show later in Sect. 2.1.

Multiple analysis pipelines are used by GES to produce stellar parameters and chemical abundances. The working group (WG) that takes care of FGK-type UVES spectra is WG11, which is split into different nodes producing independent results (Smiljanic et al. 2014). The UVES spectrum reduction is centralized (see Sacco et al. 2014, for a description) and each node 
is provided with one-dimensional (1D), wavelength calibrated, and sky subtracted spectra. Up to 11 nodes produced UVES results for iDR4. They have in common the stellar atmospheres (1D MARCS, Gustafsson et al. 2008), the line list (Ruffoni et al. 2014; Heiter et al. 2015), and the solar reference abundances (Grevesse et al. 2007); see Pancino et al. (2017) for the calibration strategy. The node results are homogenized by WG11 and subsequently by WG15 (see e.g. Casey et al. 2016, for more details), producing the recommended parameters and chemical abundances released first internally and then to the public. We use here the internal data release 4 (iDR4) which contains 54490 stars observed up to the end of June 2014 (i.e. 30 months); the public version, released after a further validation process, can be accessed through the ESO Phase 3 webpage ${ }^{2}$.

\subsection{Sample selection}

To investigate the Galactic lithium enrichment history we select well-measured main sequence field stars with UVES spectra from the GES iDR4 catalogue. In our selection, 1884 UVES stars are marked as field stars, including those of the Galactic disc and halo designated as MW $\left(G E \_M W\right)$ fields, standard CoRoT ( $G E \_S D \_C R$ ) field, standard radial velocity $\left(G E \_S D \_R V\right)$ field, and stars to the Galactic Bulge direction $\left(G E_{-} M W_{-} B L\right)$. We note that these classifications are also used in the public GES data releases. We set the surface gravity $\log (g)=3.7$ dex as the criterion to separate the dwarf and giant stars; Fig. 1 illustrates the division. The dwarfs largely outnumber the interloping giants (in our field UVES sample we have 1524 dwarfs and 360 giants, respectively), which indicates that the GES target selection was successful.

We exclude the evolved giant stars in this study because their $\mathrm{Li}$ abundances are altered by internal mixing processes and no longer reflect the Galactic Li enrichment history. When stars experience their first dredge-up after leaving the main sequence, the surface convective zone deepens and brings materials from the hot interior to the stellar surface. Lithium, which is easily destroyed at several million Kelvin, has its abundance significantly decreased at this stage because of both dilution and destruction. In fact, we also use this $\mathrm{Li}$ abundance drop to ensure that our dwarf-giant separation at $\log (g)=3.7$ is reliable (see the left panel of Fig. 1). We note that there are several giants in this Figure that do not follow the $A(\mathrm{Li})-\log (g)$ trend; these stars have already been discussed by Casey et al. (2016) who report on the "Li-rich giant problem" using GES data. Compared to the giant stars, main sequence stars with the same stellar mass have a much thinner surface convective zone, which could save Li from destruction. In this paper we focus on these main sequence stars with $\log (g) \geq 3.7$ dex.

However, for main sequence stars, another long-term stellar process, microscopic diffusion, could also lead to a depletion of the surface elements as the star ages (see e.g. Richard et al. 2002; Korn et al. 2007; Xiong \& Deng 2009; Nordlander et al. 2012; Fu et al. 2015; Dotter et al. 2017). To ensure that different ages of our sample stars do not introduce a significant dispersion in $\mathrm{Li}$ abundance, we check the pure effect of microscopic diffusion using the stellar model code PARSEC (V1.2s, Bressan et al. 2012). Three kinds of microscopic diffusion, including pressure diffusion, temperature diffusion, and concentration diffusion, as described in Thoul et al. (1994), are considered. To simulate the pure effect of microscopic diffusion, neither extra mixing (e.g. envelope overshooting) nor pre-main

\footnotetext{
2 http://www.eso.org/qi/
}

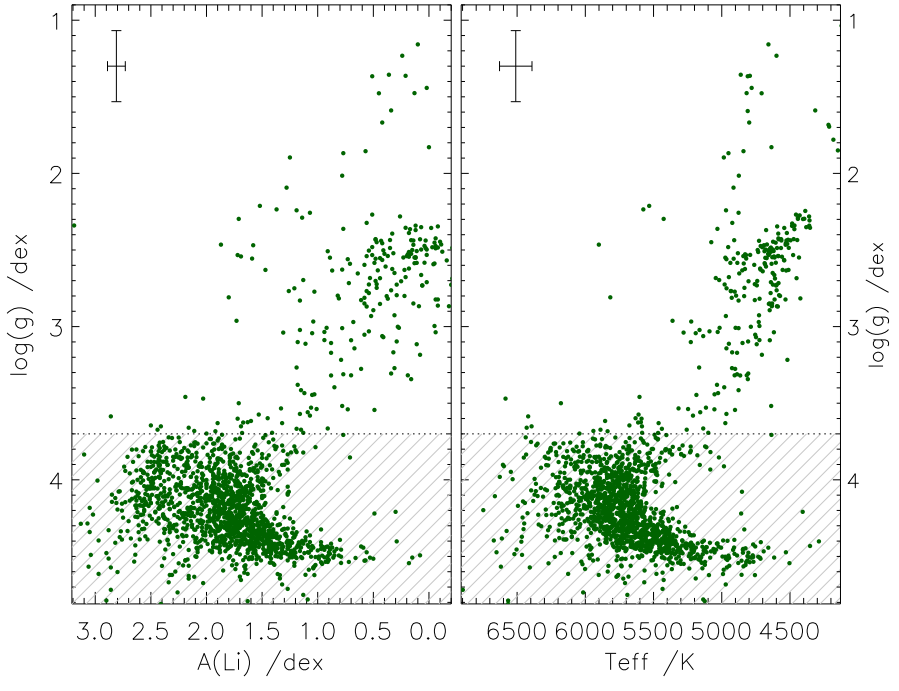

Fig. 1. Field stars in GES iDR4 catalogue with UVES observations. The left panel displays the Li content (LTE) against $\log (g)$, and the right panel shows $\log (g)$, as an indicator of the evolutionary phase, against effective temperature $\left(T_{\text {eff }}\right)$. We consider stars with $\log (g) \geq 3.7$ dex, namely those falling in the shaded areas, as main sequence stars. The median values of the parameter error are displayed in the left upper corner of each panel.

sequence accretion is applied in the models. In Fig. 2 three solar metallicity stars with different masses are shown as examples. Starting from the meteorites' Li abundance, the main Li destruction in these stars occurs early in stellar life (see also Chen et al. 2001). During their main sequence phase Li abundances (solid lines) decrease $\sim 0.2$ dex. Their temperatures at the bottom of the surface convective zone ( $T_{\text {bot }}$, dotted lines), which is an indicator of the rate of Li burning, are so low $\left(1.3 \sim 2.5 \times 10^{6} \mathrm{~K}\right)$ that even 7-10 Gyr of evolution cannot lead to a notable destruction. Thus Li depletion from nuclear reaction is negligible in these stars during the main sequence phase; the main depletion comes from the microscopic diffusion. Microscopic diffusion takes $7-$ 10 Gyr to decrease the surface $\mathrm{Li}$ abundance by $\sim 0.2$ dex, which is the typical Li measurement uncertainty of our sample. If extra mixing, which slows the diffusion (Xiong \& Deng 2009), is considered, the depletion time will be even longer. Therefore, even a 7-10 Gyr age difference of the Galactic field stars will not affect our investigation. For stars with lower mass, and thus a deeper surface convective zone (e.g. $M=0.90 M_{\odot}$ star in Fig. 2), their Li depletion can be traced by $T_{\text {eff }}$; we discuss this in detail in Sect. 3.

We then exclude stars with large $[\mathrm{Fe} / \mathrm{H}]$ errors. Figure 3 shows the distribution of $[\mathrm{Fe} / \mathrm{H}]$ error for the 1524 main sequence field UVES stars; those with $[\mathrm{Fe} / \mathrm{H}]$ error $<0.13$ dex (95\% of the sample) are selected as "well-measured" objects. Some of the well-measured stars are members of multiple stellar system, or have emission lines in their spectra. Since multiple stellar system members carve up their initial Li (e.g. in binary stars the primary one has higher $\mathrm{Li}$ abundance, González Hernández et al. 2008; Aoki et al. 2012; Fu et al. 2015) and the emission lines are likely from pre-main sequence stars which may undergo Li depletion, we remove these stars from our sample to avoid confusion. This reduces the sample to 1432 objects. In GES iDR4, 1097 of them are labelled as stars with Li upper limits, and 335 as true measurements. We double check the spectra of stars with labelled Li measurements and mark 302 of them as high quality spectra around the 


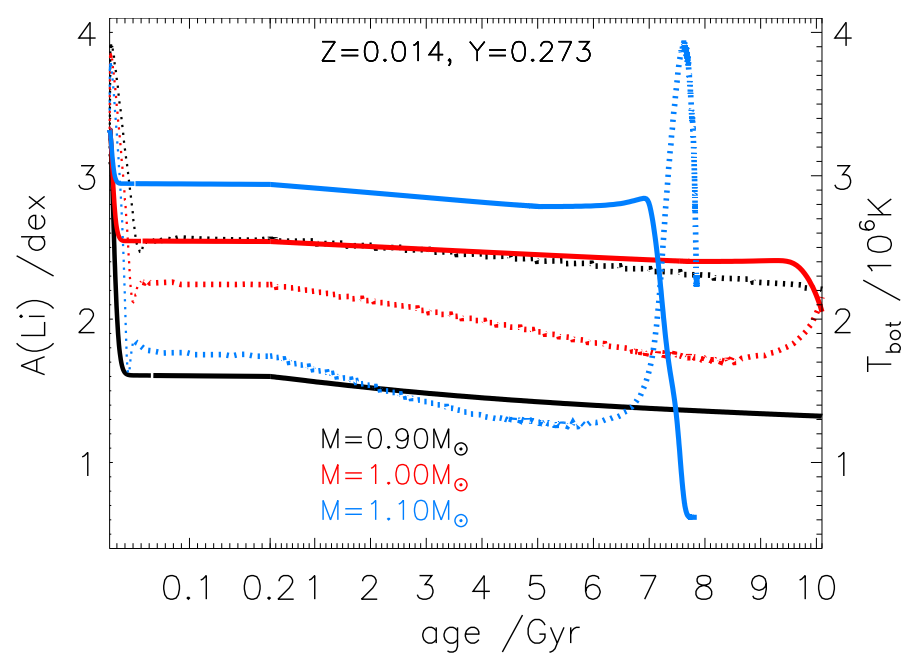

Fig. 2. Pure effect of microscopic diffusion on Li abundance during the first $10 \mathrm{Gyr}$ from stellar model PARSEC. Neither envelope overshooting nor pre-main sequence accretion is applied. Li abundances (solid lines) and the temperature at the bottom of surface convective zone ( $T_{\text {bot }}$, dotted lines) are displayed as a function of stellar age. Different colours indicate different stellar mass as indicated in the legend.

Li resonance line. In our final sample we thus have 1399 wellmeasured UVES main sequence field stars sorted into two categories: i) labelled Li upper limits (1097 stars, with $\mathrm{S} / \mathrm{N}$ ranging in 12-259 and median $S / N=63)$; and ii) checked Li measurements (302 stars, $\mathrm{S} / \mathrm{N}$ from 18 to 319 , and median $S / N=83$ ).

\section{Results}

The abundance analysis of Li in GES iDR4 is based on the local thermodynamic equilibrium (LTE) assumption, however in reality the line formation in stellar atmosphere is a more complex process, and non-LTE (NLTE) effect should be accounted for. We apply the NLTE corrections to the Li I $\lambda=6708 \AA$ line using the correction grid from Lind et al. (2009). Figure 4 displays the correction departures $\left(A_{\mathrm{Li}}^{\mathrm{NLTE}}-A_{\mathrm{Li}}^{\mathrm{LTE}}\right)$ as a function of each parameter $\left(A_{\mathrm{Li}}^{\mathrm{LTE}}, T_{\mathrm{eff}},[\mathrm{Fe} / \mathrm{H}], \log (g)\right.$, and micro-turbulence $\left.\xi\right)$. We calculate also the NLTE correction uncertainties introduced by the errors of these parameters. The median values of the uncertainties are $\sim 0.02-0.035$ dex from each parameter, which are negligible. The overall NLTE corrections, as seen from Fig. 4, are not significant for stars in our sample; even the largest departure is less than or equal to the value of the typical $\mathrm{Li}$ abundance error ( 0.2 dex) in LTE. Table 1 lists the LTE and NLTE results, together with other chemical abundances and the stellar parameters that we use in this study. Hereafter we use $A_{\mathrm{Li}}$ to represent $A_{\mathrm{Li}}^{\mathrm{NLTE}}$.

In Fig. 5 we present the general behaviours of $A_{\mathrm{Li}}$ against $T_{\text {eff }}, \log (g)$, and $[\mathrm{Fe} / \mathrm{H}]$, respectively. Cooler stars show lower $\mathrm{Li}$ abundances as expected (see the left panel) because they have a deeper surface convective zone which burns Li. The coolest stars with Li upper limits are also those that have larger $\log (g)$ (see the central panel). They are lower main sequence stars with small stellar mass since $\log (g)$ is an indicator of the evolutionary phase. The upper envelope of the Li evolution with $[\mathrm{Fe} / \mathrm{H}]$ (see the right panel of Fig. 5) is traditionally believed to reflect the Li enrichment history of the Galactic ISM, taking $[\mathrm{Fe} / \mathrm{H}]$ as an index of the total metallicity (see Abia et al. 1998; Romano et al. 1999, 2001; Travaglio et al. 2001; Prantzos 2012). 


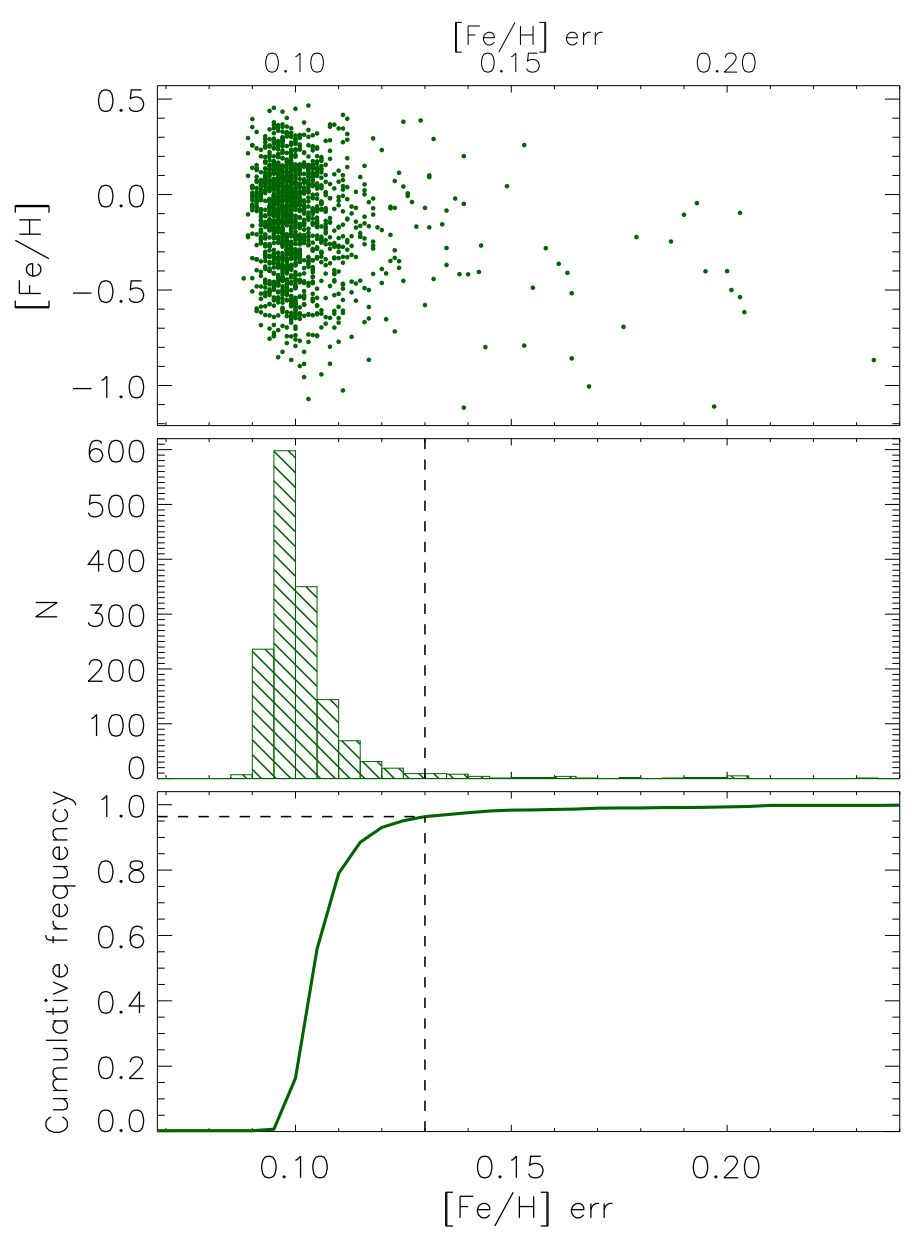

Fig. 3. Distribution of $[\mathrm{Fe} / \mathrm{H}]$ error for main sequence field UVES stars in GES iDR4 catalogue. The upper panel displays the relation of $[\mathrm{Fe} / \mathrm{H}]$ and $[\mathrm{Fe} / \mathrm{H}]$ error, while the centre and lower panels show the histogram and cumulative frequency of $[\mathrm{Fe} / \mathrm{H}]$ error, respectively. Ninety-five percent of stars lie in the interval smaller than 0.13 dex (vertical dashed lines in the last two panels).

In the following sections we combine our $\mathrm{Li}$ abundances with $[\alpha / \mathrm{Fe}],[\mathrm{Ba} / \mathrm{Fe}]$, and $[\mathrm{Y} / \mathrm{Fe}]$ ratios to shed new light on the $\mathrm{Li}$ enrichment histories of different Galactic disc components.

\subsection{Distinction between the Galactic thick and thin discs}

A precise determination of the Galactic thick and thin disc membership requires a detailed knowledge of the space motions, which are calculated from the stellar distances, proper motions and radial velocities. Unfortunately only nine stars in our sample have this information from Gaia DR1 TGAS catalogue (Michalik et al. 2015; Gaia Collaboration et al. 2016). We are looking forward to Gaia DR2, which will be released in spring 2018 and will provide the five-parameter astrometric solutions and help us to draw a precise map of the thick and thin discs for almost the entire Gaia catalogue.

The abundance of $\alpha$ elements relative to iron $([\alpha / \mathrm{Fe}])$ are often used to chemically separate the Galactic thick disc from thin disc stars when the space motion information is lacking (e.g. Fuhrmann 1998; Gratton et al. 2000; Reddy et al. 2003; Venn et al. 2004; Bensby et al. 2005; Rojas-Arriagada et al. 2017). The $\alpha$ elements (O, Ne, Mg, Si, S, Ar, Ca, and Ti) are mostly produced by core collapse SNe (mainly Type II SNe) on short time scales, while iron is also synthesised in Type Ia SNe on longer time scales (after at least one white dwarf has been formed). Stars formed shortly after the ISM has been enriched by SNe II have enhanced $[\alpha / \mathrm{Fe}]$ ratios, while those formed sometime after SNe Ia have contributed most of the Fe have higher iron abundances and lower $[\alpha / \mathrm{Fe}]$ ratios. Thus the $[\alpha / \mathrm{Fe}]$ ratio is a cosmic-clock, or better, it echoes the formation history (see e.g. Tinsley 1979; Matteucci \& Greggio 1986; Haywood et al. 2013, and the references therein). The Galactic thick disc stars usually have lower $[\mathrm{Fe} / \mathrm{H}]$ values and higher $[\alpha / \mathrm{Fe}]$, while most of the thin disc stars tend to have higher iron abundance and lower $[\alpha / \mathrm{Fe}]$ values. However the criterion adopted for separation differs in different works.

We define $[\alpha / \mathrm{Fe}]$ ratios for the stars in our sample, taking into account four $\alpha$ elements $(\mathrm{Mg}, \mathrm{Ca}, \mathrm{Si}$, and $\mathrm{Ti})$ :

$$
\begin{aligned}
& n(\alpha)=n(\mathrm{Mg} \mathrm{I})+n(\mathrm{Ca} \mathrm{I})+n(\mathrm{Si} \mathrm{I})+n(\mathrm{Ti} \mathrm{I})+n(\mathrm{Ti} \mathrm{II}), \\
& {[\alpha / \mathrm{Fe}]=\log \left(\frac{n(\alpha)}{n(\mathrm{Fe})}\right)_{*}-\log \left(\frac{n(\alpha)}{n(\mathrm{Fe})}\right)_{\odot} .}
\end{aligned}
$$

The other elements (and isotopes) are not included because they are not measured in all our sample stars. By taking into account the measurement uncertainties of the six parameters in Eqs. (1) and (2) (A(Mg I), A(Si), A(Ca I), A(Ti I), A(Ti II), and $[\mathrm{Fe} / \mathrm{H}])$, we derive the mean value of $[\alpha / \mathrm{Fe}]$ and its corresponding $1 \sigma$ uncertainty using the Markov chain Monte Carlo (MCMC) code "emcee" (Foreman-Mackey et al. 2013). After a burn-in phase of 100 steps to ensure that the chains have converged, we performed 150 MCMC steps for each star. The final results are listed in Table 1.

Here we perform a tentative separation based on $[\alpha / \mathrm{Fe}]$. We adopt a separation method similar to Recio-Blanco et al. (2014) and Mikolaitis et al. (2014) that divides the sample stars into several $[\mathrm{Fe} / \mathrm{H}]([\mathrm{M} / \mathrm{H}]$ in the case of Recio-Blanco et al. 2014; Mikolaitis et al. 2014) bins and finds the possible $[\alpha / \mathrm{Fe}]$ demarcation between the thick and thin discs in each interval. Our resulting tentative division is consistent with the separation proposed by Adibekyan et al. (2012) who separate the highand low- $\alpha$ stars with very high-resolution and high- $\mathrm{S} / \mathrm{N}$ data. In Fig. 6 we show the separation for all sample stars, the division for stars with $\mathrm{Li}$ measurements, and those with $\mathrm{Li}$ upper limits are also displayed. There are 569 stars in our sample possibly belonging to the thick disc (73 of them have $\mathrm{Li}$ measurements and 496 are stars with Li upper limits) and 830 stars that are likely thin disc members (229 stars are from the category of Li measurements and 601 stars have Li upper limits). Our tentative separation is essentially similar to the divisions adopted for slightly more metal-poor stars in GES data by Recio-Blanco et al. (2014); Mikolaitis et al. (2014) in the $[\alpha / \mathrm{Fe}]-[\mathrm{M} / \mathrm{H}]$ and $[\mathrm{Mg} / \mathrm{M}]-[\mathrm{M} / \mathrm{H}]$ planes. We choose not to adopt their divisions because they are based on GIRAFFE spectra and a previous data release.

Figure 7 shows the behaviour of Li enrichment in the two discs, when our sample stars are divided according to the criterion outlined above. All stars with $A_{\mathrm{Li}}>2.2$ dex (the Spite Plateau value) are Li-enriched compared to Pop. II stars. Since we are not able to precisely disentangle thick from thin disc stars in our sample without the space motion information, we cannot simply select the highest $A_{\mathrm{Li}}$ as the initial Li value. Therefore, we adopt the method used in Lambert \& Reddy (2004); Delgado Mena et al. (2015); Guiglion et al. (2016) that selects the six stars with the highest $A_{\mathrm{Li}}$ in each $[\mathrm{Fe} / \mathrm{H}]$ bin and calculates their mean $A_{\mathrm{Li}}$ values (weighted by the reciprocal of $\mathrm{Li}$ abundance errors in LTE) to track the trend of Li enrichment; the standard deviations of their $A_{\mathrm{Li}}$ is considered to be 

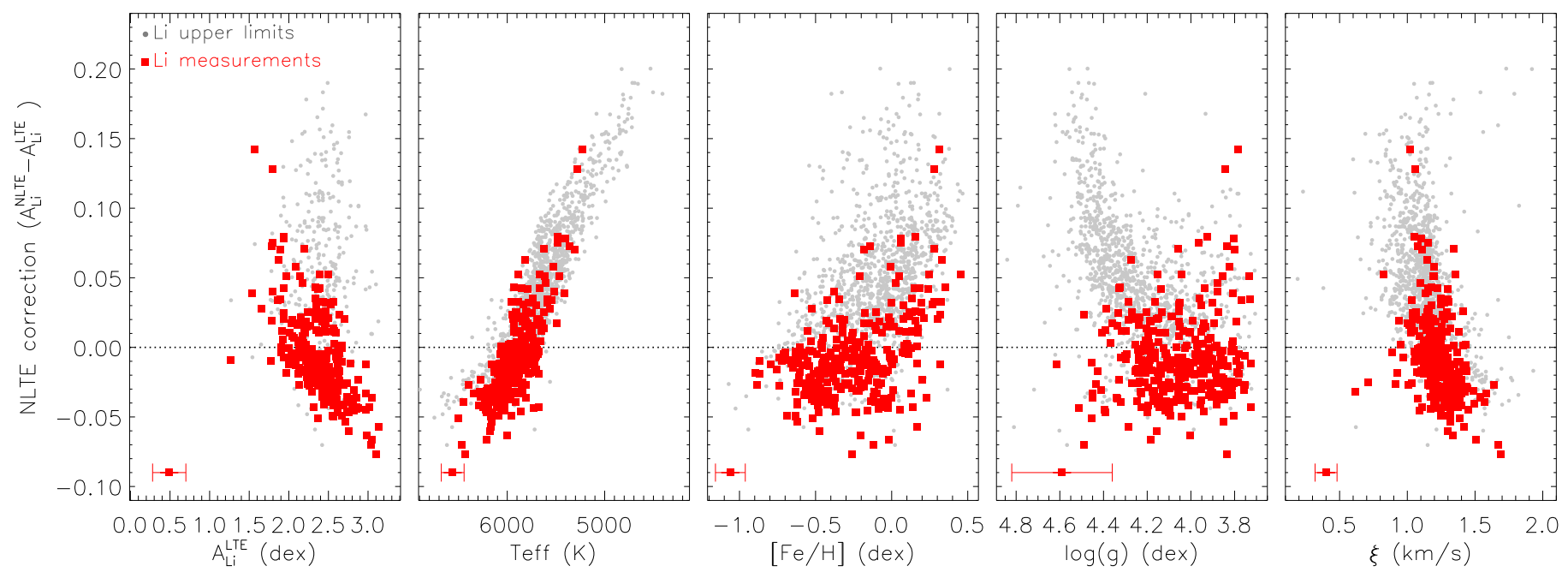

Fig. 4. NLTE Li abundances corrections vs. various parameters. The filled red squares are stars with checked Li measurements (302 stars), and grey dots represent those labelled with Li upper limits in GES iDR4 catalogue (1097 stars). The median error of each parameter is displayed in the lower-left corner of each panel.
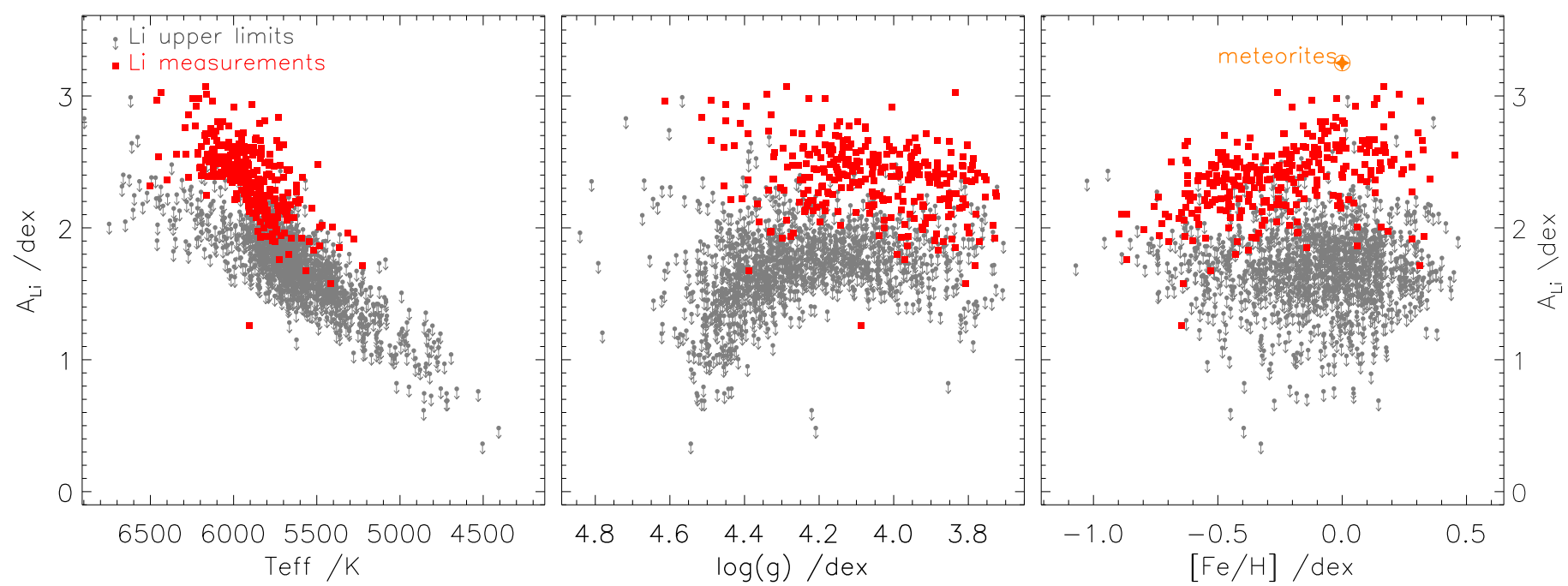

Fig. 5. NLTE Li abundance for stars in our final sample as a function of $T_{\text {eff }}(l e f t), \log (g)$ (centre), and $[\mathrm{Fe} / \mathrm{H}]$ (right), respectively. In all three panels, filled red squares are the stars with checked Li measurements (302 stars), and grey dots represent those labelled with Li upper limits in GES iDR4 catalogue (1097 stars). The Li abundance of meteorites is also marked in the right panel.
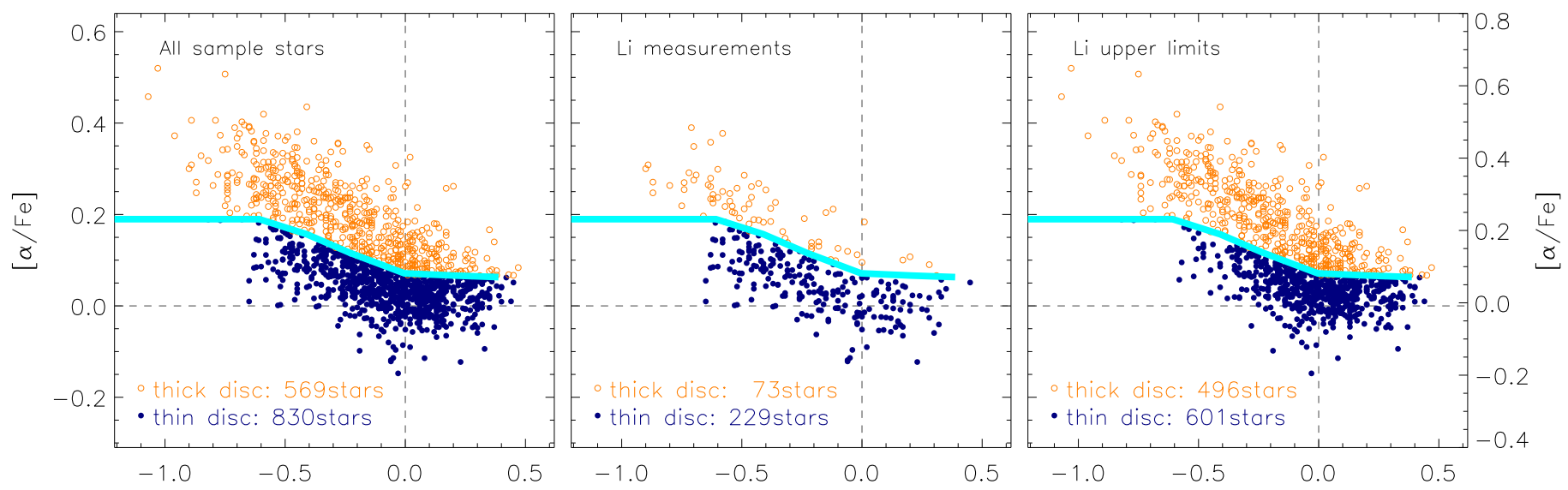

Fig. 6. Tentative separation between thick and thin discs in the $[\alpha / \mathrm{Fe}]-[\mathrm{Fe} / \mathrm{H}]$ plane for all sample stars (left panel), stars with Li measurements (central panel), and stars with Li upper limits (right panel). In all panels the vertical and horizontal dashed lines indicate solar values. Filled blue dots represent the Galactic thin disc stars while open orange circles are the thick disc objects. The cyan line shows the division proposed by (Adibekyan et al. 2012). 


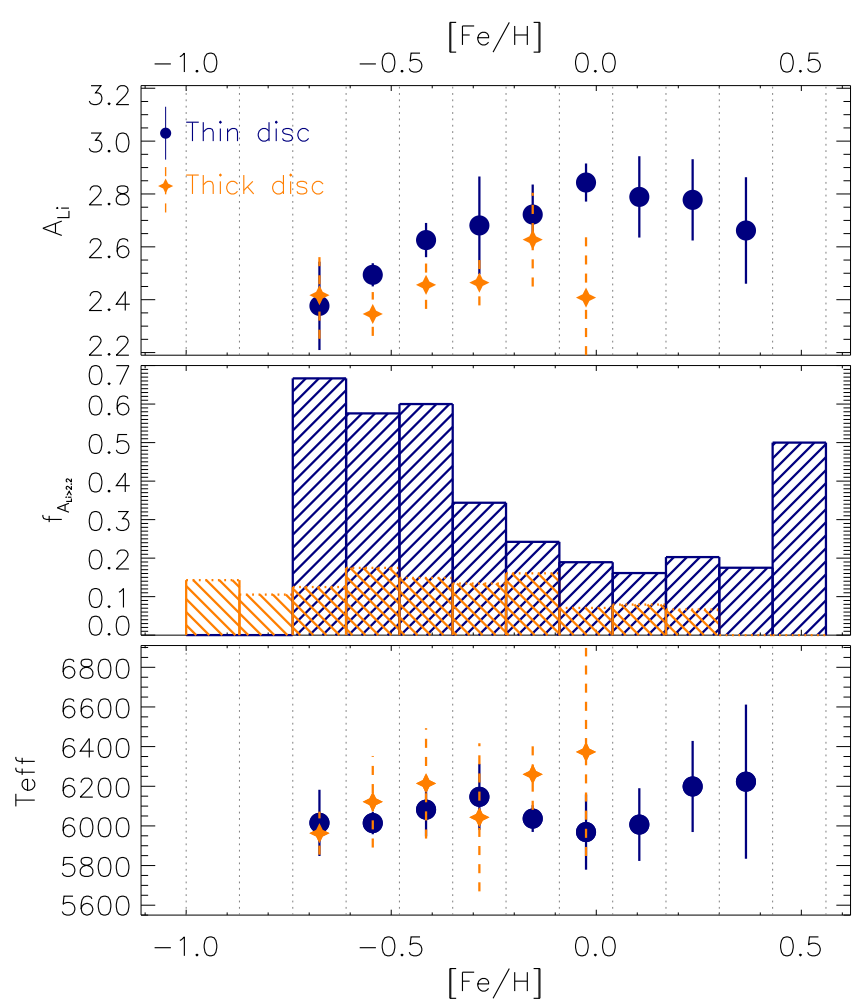

Fig. 7. Stars with enriched $\mathrm{Li}\left(A_{\mathrm{Li}}>2.2 \mathrm{dex}\right)$. Upper panel: errorweighted mean $A_{\mathrm{Li}}$ values of the six stars with the highest $\mathrm{Li}$ abundance in each $[\mathrm{Fe} / \mathrm{H}]$ bin; the standard deviation of their $A_{\mathrm{Li}}$ values is considered as the uncertainty. Blue filled dots represent the thin disc and the orange stars indicate the thick disc. The symbols are displayed when there are more than six Li-enriched thin/thick stars in the bin. Middle panel: fractions of Li-enriched stars $\left(f_{A(\mathrm{~L} i)>2.2}\right)$ in each $[\mathrm{Fe} / \mathrm{H}]$ interval for thin disc stars (blue-shades histogram) and thick disc stars (orangeshaded histogram). Lower panel: error-weighted mean $T_{\text {eff }}$ values of the six stars with the highest $\mathrm{Li}$ abundance in each $[\mathrm{Fe} / \mathrm{H}]$ bin; the standard deviation of their $T_{\mathrm{eff}}$ values is considered as the uncertainty. Symbols are the same as in the upper panel.

the corresponding uncertainty. In the upper panel of Fig. 7 the blue filled dots and the orange stars display these trends for the Galactic thin disc and thick disc, respectively. Our Li trend of thin disc stars is systematically lower than the one proposed by Guiglion et al. (2016), while it is compatible with the overall trend of Delgado Mena et al. (2015). This may be because of sample selection effects. Strictly speaking, the trends of main sequence mean $A_{\mathrm{Li}}$ is a lower limit to the real $\mathrm{Li}$ evolution in both the thin and thick disc. Some depletion (although perhaps little) has had to happen in both groups of stars. The fraction of the Li-enriched stars in each $[\mathrm{Fe} / \mathrm{H}]$ interval represents the overall level of $\mathrm{Li}$ enrichment. In the middle panel of Fig. 7 we present the fraction of Li-enriched stars in the thin (blue histogram) and thick (orange histogram) discs. The thin disc has much higher Lienriched star fractions compared to the thick disc. We perform a K-S test to compare the two distributions, the maximum deviation between the cumulative distribution of the two histograms is $D=0.75$, and the significance level of the K-S statistic is 0.0009. As mentioned in Sect. 2.1, $T_{\text {eff }}$ is a key parameter to trace the stellar Li destruction (stars with lower $T_{\text {eff }}$ have lower $\left.A_{\mathrm{Li}}\right)$. In order to investigate whether thick disc stars have experienced a stronger Li destruction than the thin disc stars, we compare the mean $T_{\text {eff }}$ (weighted by the reciprocal of $T_{\text {eff }}$ errors) of the six stars with highest $A_{\mathrm{Li}}$ in each $[\mathrm{Fe} / \mathrm{H}]$ bin, and take their $T_{\text {eff }}$ standard deviation as the uncertainty; results are displayed in

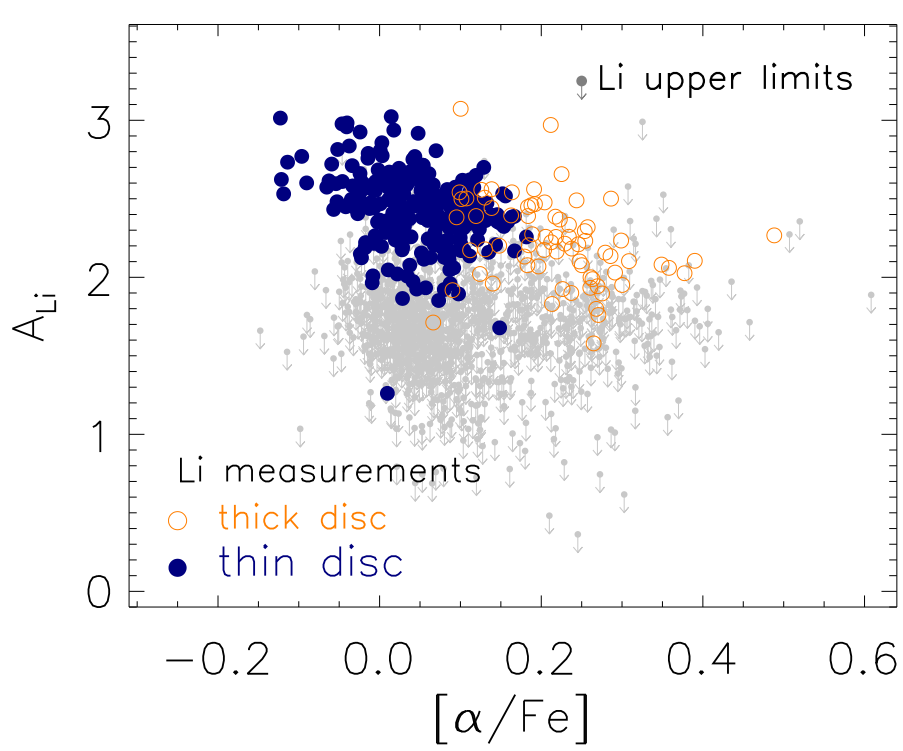

Fig. 8. NLTE Li abundance verses $[\alpha / \mathrm{Fe}]$. Stars with Li measurements are separated as thin (filled blue dots) and thick (open orange circles) disc stars, the same as in the middle panel of Fig. 6. The grey dots represent sample stars with Li upper limits.

the lower panel of Fig. 7. It becomes apparent that the thick disc stars do not show a lower $T_{\text {eff }}$ compared to the thin disc stars, in some of the $[\mathrm{Fe} / \mathrm{H}]$ bins they even have higher $T_{\text {eff }}$ than the thin disc ones though their $A_{\mathrm{Li}}$ is lower as shown in the upper panel of the figure. As seen in all the three panels of Fig. 7, we conclude that the Galactic thin disc has higher $A_{\mathrm{Li}}$ and higher overall level of $\mathrm{Li}$ enrichment than the thick disc, the $\mathrm{Li}$ trend difference between the thick and thin discs is not due to the stellar destruction, but reflects different initial $\mathrm{Li}$ abundances of the two discs.

\section{2. $\mathrm{Li}-[\alpha / \mathrm{Fe}]$ anticorrelation}

In addition to the tentative thick/thin disc separation, we investigate the relation between $A_{\mathrm{Li}}$ and $[\alpha / \mathrm{Fe}]$, especially for stars with actual Li measurements, in order to gain a deeper insight into the $\mathrm{Li}$ enrichment histories of the Galactic thick and thin disc.

In Fig. 8 we display the overall relation between $A_{\mathrm{Li}}$ and $[\alpha / \mathrm{Fe}]$. A Li- $[\alpha / \mathrm{Fe}]$ anticorrelation is clearly seen in this figure and is highly statistically significant (its Pearson's correlation for stars with Li measurements has a confidence level $>99 \%$ ). However one cannot ignore that as $[\mathrm{Fe} / \mathrm{H}]$ increases, $A_{\mathrm{Li}}$ rises (see the right panel of Fig. 5) while $[\alpha / \mathrm{Fe}]$ decreases (see Fig. 6). To eliminate the $[\mathrm{Fe} / \mathrm{H}]$ evolutionary effect in the $\mathrm{Li}-[\alpha / \mathrm{Fe}]$ anticorrelation, one should compare stars with similar $[\mathrm{Fe} / \mathrm{H}]$ values. For this purpose we present $A_{\mathrm{Li}}$ as a function of $[\alpha / \mathrm{Fe}]$ in different $[\mathrm{Fe} / \mathrm{H}]$ bins in Fig. 9. The bin size is $0.26 \mathrm{dex}$, which is twice the maximum error on $[\mathrm{Fe} / \mathrm{H}]$ for our sample stars. To avoid the bin selection bias, 0.13 dex is overlapped between the adjacent $[\mathrm{Fe} / \mathrm{H}]$ bins. We calculate the mean values of Pearson's correlation coefficient $\left(R_{0}(P)\right)$ and its significance level $P_{0}$ between $A_{\mathrm{Li}}$ and $[\alpha / \mathrm{Fe}]$ in each $[\mathrm{Fe} / \mathrm{H}]$ bin. We also derive the possibility of the anticorrelation in each bin taking into account the abundance uncertainties. In this case, $\mathrm{Li}$ abundance error in LTE measurement is considered as the uncertainty of NLTE results, and a Bayesian linear regression method described in Kelly (2007) is performed. If the number of stars in the bin is greater than 10 , 

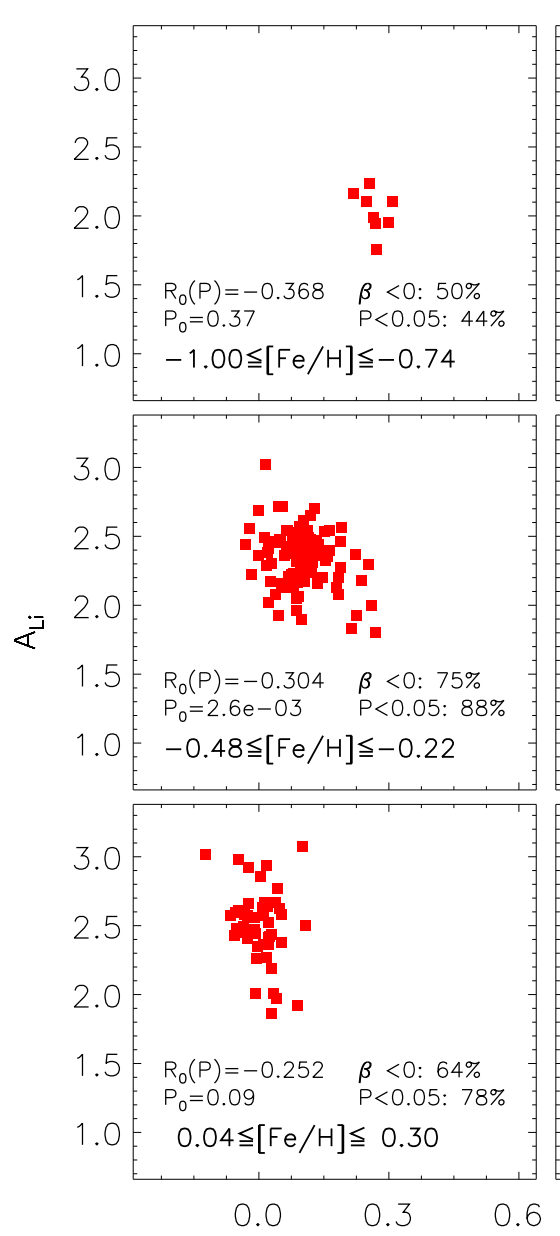
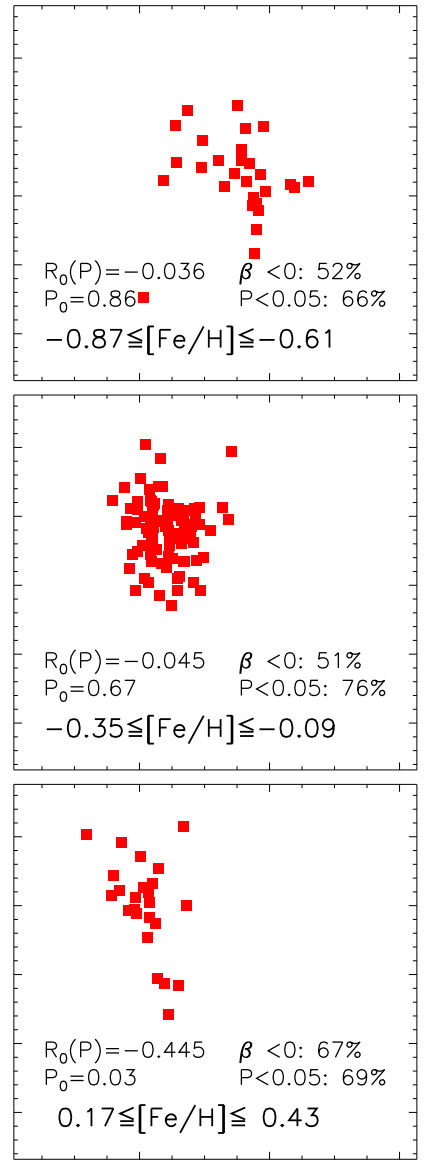

$0.0 \quad 0.3 \quad 0.6$
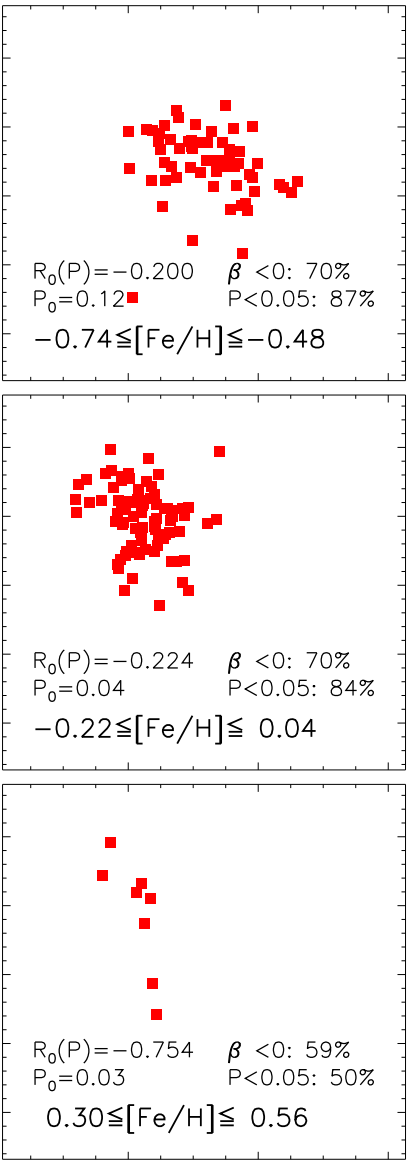
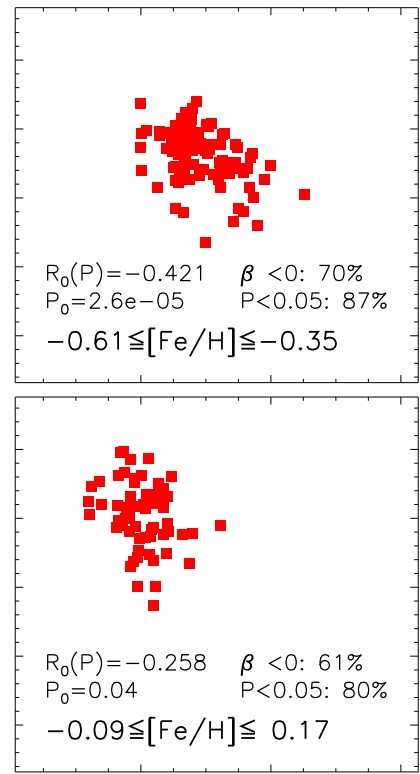

$$
[\alpha / \mathrm{Fe}]
$$

Fig. 9. NLTE Li abundance as a function of $[\alpha / \mathrm{Fe}]$ in each $[\mathrm{Fe} / \mathrm{H}]$ bin for our sample stars with Li measurements. The range of $[\mathrm{Fe} / \mathrm{H}]$ is specified in each plot. The $[\mathrm{Fe} / \mathrm{H}]$ bin size is 0.26 dex. In each bin we label the mean correlation coefficient $\left(R_{0}(P)\right)$ between $A_{\mathrm{Li}}$ and $[\alpha / \mathrm{Fe}]$, the mean correlation significance level $\left(P_{0}\right)$, the possibility of $\mathrm{Li}-[\alpha / \mathrm{Fe}]$ anticorrelation $(\beta<0)$ if considering the abundance uncertainties, and the possibility of $P<0.05$ for the anticorrelation.

then the Markov chains will be created using the Gibbs sampler; otherwise the Metropolis-Hastings algorithm is used. A negative value of the linear regression slope $\beta$ means anticorrelation. For each $[\mathrm{Fe} / \mathrm{H}]$ bin, we calculate the possibility of an anticorrelation $(\beta<0)$ of $\mathrm{Li}-[\alpha / \mathrm{Fe}]$, and find that the possibility is $\gtrsim 68 \%(1 \sigma)$ for half of them, with a corresponding 1-2 $\sigma$ significance of $P<0.05$ (i.e. the confidence interval is greater than $95 \%)$. This means that, at similar $[\mathrm{Fe} / \mathrm{H}], A_{\mathrm{Li}}$ is very likely anti correlated with $[\alpha / \mathrm{Fe}]$ for field stars.

We have shown in Fig. 5 that $A_{\mathrm{Li}}$ decreases with lower $T_{\text {eff }}$, as $T_{\text {eff }}$ is a tracer of the Li destruction. In Fig. 10 we show it is highly probable that stars with similar $T_{\text {eff }}$ have their Li abundances anti-correlated with $[\alpha / \mathrm{Fe}]$. Since $98 \%$ of our sample stars have $T_{\text {eff }}$ error less than $150 \mathrm{~K}$, we choose this value as the bin size in Fig. 10. To avoid the bin selection bias we overlap $75 \mathrm{~K}$ between the adjacent $T_{\text {eff }}$ bins. The anticorrelation between the mean values of $A_{\mathrm{Li}}$ and $[\alpha / \mathrm{Fe}]$ is significant at the level of $P \leq 0.05$, except for the intervals with too few stars (the first three and last two bins). If the abundance uncertainties are considered, we use the same Bayesian linear regression method as in Fig. 8 to derive the $\mathrm{Li}-[\alpha / \mathrm{Fe}]$ anticorrelation possibility and its corresponding significance level; results are listed in Fig. 10.

It is of great interest to compare the correlation between $A_{\mathrm{Li}}$ and $[\alpha / \mathrm{Fe}]$ for stars with similar evolutionary phase (position in the main sequence) as well. We use $\log (g)$ as an index of the evolutionary phase and present the $\mathrm{Li}-[\alpha / \mathrm{Fe}]$ anticorrelation in Fig. 11. The $\log (g)$ bin size is $0.235 \mathrm{dex}$, and is based on a criterion on the $\log (g)$ error that covers $68 \%$ of the sample stars. Similar to our treatment for $T_{\text {eff }}$ and $[\mathrm{Fe} / \mathrm{H}]$, we overlap half of the bin size between the adjacent bins to avoid selection bias. In each $\log (g)$ interval the mean $A_{\mathrm{Li}}-[\alpha / \mathrm{Fe}]$ correlation is negative, and the mean correlation significance levels $\left(P_{0}\right)$ are less than 0.05 except in the first bin which has only a few stars. When taking into account the abundance uncertainties as described above for different $T_{\text {eff }}$ and $[\mathrm{Fe} / \mathrm{H}]$ bins, there is a (very) high possibility for the $\mathrm{Li}-[\alpha / \mathrm{Fe}]$ anticorrelation in almost every $\log (g)$ bin.

To ensure that the $\mathrm{Li}-[\alpha / \mathrm{Fe}]$ anticorrelation echoes the initial $A_{\mathrm{Li}}$ and is not affected by any stellar evolution effect, we check the behaviour of $A(\mathrm{Li})$ in stellar evolution models. We use the latest version of PARSEC (Bressan et al. 2012; Fu et al. 2017) to calculate Li evolution for two stars with the same metallicity, helium content, stellar mass, and initial Li abundance; one star, however, has $[\alpha / \mathrm{Fe}]=0.4 \mathrm{dex}$ and the other $[\alpha / \mathrm{Fe}]=0$ (solar-scaled). Though the star with $[\alpha / \mathrm{Fe}]=0.4$ dex is hotter in every evolutionary phase (see the left panel of Fig. 12), there is no measurable difference between its $A_{\mathrm{Li}}$ and that of the star with $[\alpha / \mathrm{Fe}]=0$, neither at the same age (see the central panel) nor at the same $\log (g)$. The different $A_{\mathrm{Li}}$ behaviour in stars with 

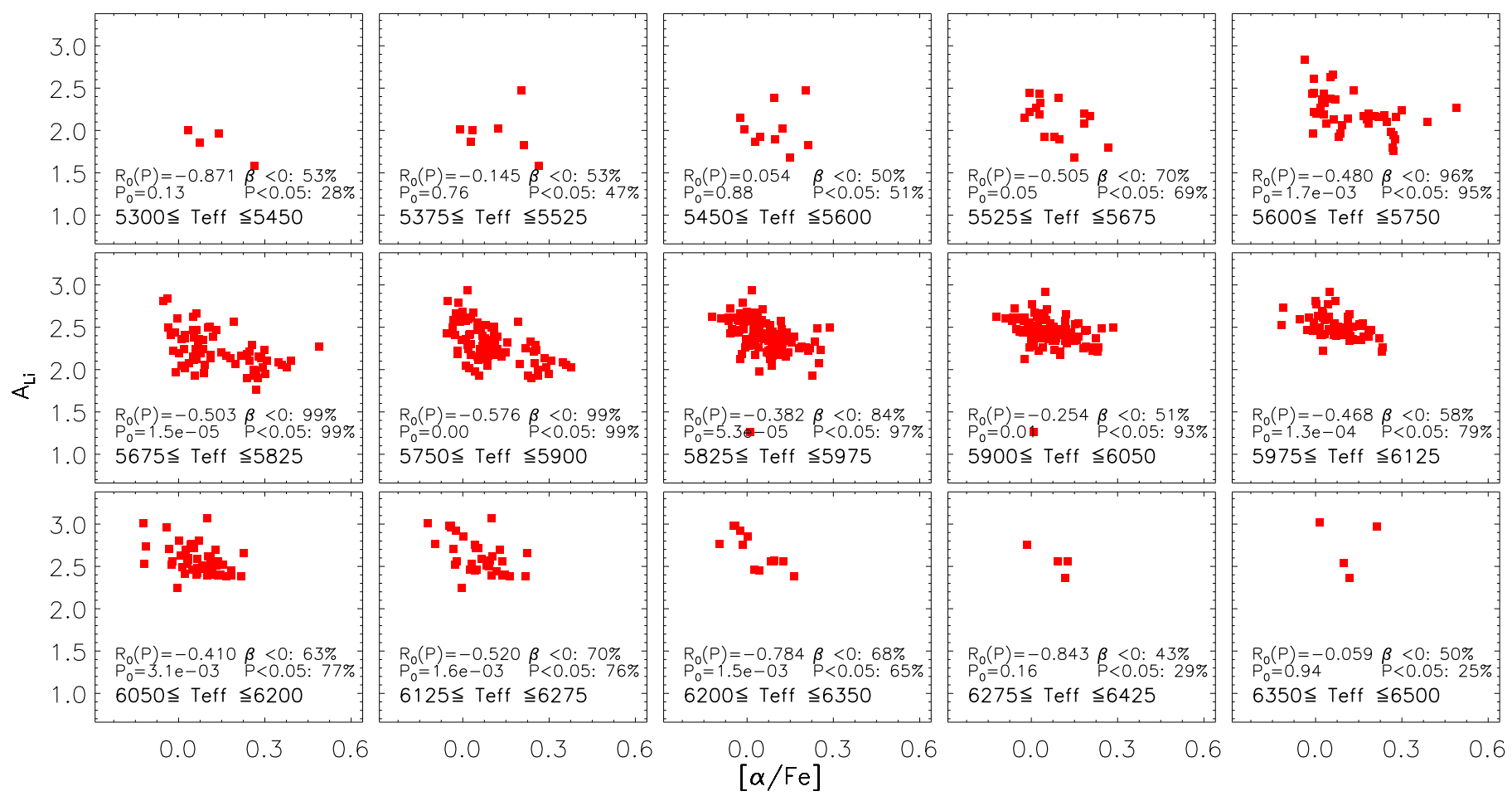

Fig. 10. NLTE Li abundance as a function of $[\alpha / \mathrm{Fe}]$ in each $T_{\text {eff }}$ bin for our sample stars with Li measurements. The range of $T_{\text {eff }}$ is specified in each plot, with bin size of $150 \mathrm{~K}$. Here we label the mean correlation coefficient $\left(R_{0}(P)\right)$ between $A_{\mathrm{Li}}$ and $[\alpha / \mathrm{Fe}]$, the mean correlation significance level $\left(P_{0}\right)$, the possibility of $\mathrm{Li}-[\alpha / \mathrm{Fe}]$ anticorrelation $(\beta<0)$ if consider the abundance uncertainties, and the possibility of $P<0.05$ for the anticorrelation.

different $[\alpha / \mathrm{Fe}]$ has to come from their initial $\mathrm{Li}$ abundances which reflect different $\mathrm{Li}$ enrichment histories in different disc components.

The $\mathrm{Li}-[\alpha / \mathrm{Fe}]$ anticorrelation also sheds light on the role of core collapse $\mathrm{SNe}$ in the total $\mathrm{Li}$ enrichment. Since core collapse $\mathrm{SNe}$ are the main producer of the $\alpha$ elements, if these stars are responsible also for a major $\mathrm{Li}$ production, this element should increase with $[\alpha / \mathrm{Fe}]$. Our Li- $[\alpha / \mathrm{Fe}]$ anticorrelation result instead implies that the contribution from core collapse $\mathrm{SNe}$ to the global Li enrichment is unimportant, if not negligible, thus supporting previous findings (see our Introduction).

\subsection{Li-s(-process elements) correlation}

Similarly to the $[\alpha / \mathrm{Fe}]$ ratio, the ratio of the slow $(s-)$ neutron capture process elements to iron can be regarded as a cosmic clock. $\mathrm{Ba}, \mathrm{Sr}, \mathrm{La}$, and $\mathrm{Y}$ are mainly $s$-process elements produced on long timescales by low mass AGB stars (Matteucci 2012). Since a low mass star must evolve to the AGB phase before the $s$-process can occur, the $s$-process elements are characterized by a delay in the production, much like the delay of iron production by $\mathrm{SNe}$ Ia relative to the $\alpha$ elements production by core collapse SNe. Among the four $s$-process elements mentioned above, GES provides the abundances of Y II (the first $s$-process peak element) and Ba II (the second $s$-process peak element) for all our sample stars. Their abundances behave differently in the Galactic thick and thin discs (Bensby et al. 2005, 2014; Israelian et al. 2014; Bisterzo et al. 2017; Delgado Mena et al. 2017). Unlike the Galactic thick disc stars, which show an almost constant $[\mathrm{Ba} / \mathrm{Fe}]$ abundance close to the solar value, the Galactic thin disc stars have their $[\mathrm{Ba} / \mathrm{Fe}]$ abundances increasing with $[\mathrm{Fe} / \mathrm{H}]$ and reaching their maximum values around solar metallicity, after which a clear decline is seen (see also Cristallo et al. 2015a,b, for the most recent $s$-process calculation in AGB yields). The same trend is observed in our sample. In Fig. 13 we display the $\mathrm{Li}-[\mathrm{Ba} / \mathrm{Fe}],[\mathrm{Ba} / \mathrm{Fe}]$ as a function of $[\mathrm{Fe} / \mathrm{H}]$, and the evolution of absolute $\mathrm{Ba}$ abundance $A(\mathrm{Ba})$, as derived from Ba II lines. Similar figures are also plotted for yttrium (Y II). $[\mathrm{Ba} / \mathrm{Fe}]$ and $[\mathrm{Y} / \mathrm{Fe}]$ values here are derived from MCMC simulations, taking into account the measurement uncertainties of $\mathrm{A}(\mathrm{Ba} \mathrm{II}) / \mathrm{A}(\mathrm{Y}$ II $)$ and $[\mathrm{Fe} / \mathrm{H}]$. By applying the same MCMC setups used for $[\alpha / \mathrm{Fe}]$ (see Sect. 3.1), we calculate the mean values of $[\mathrm{Ba} / \mathrm{Fe}]$ and $[\mathrm{Y} / \mathrm{Fe}]$ for each star. These values, together with their corresponding $1 \sigma$ uncertainties, are listed in Table 1 . In the literature there are several theoretical works on the evolution of $[\mathrm{Ba} / \mathrm{Fe}]$ and $[\mathrm{Y} / \mathrm{Fe}]$ in the Galactic thin disc (e.g. Pagel \& Tautvaisiene 1997; Travaglio et al. 1999, 2004; Cescutti et al. 2006; Maiorca et al. 2012; Bisterzo et al. 2017). For comparison, we show in Fig. 13 the predictions of the most recent one (Bisterzo et al. 2017) where the updated nuclear reaction network was used.

For stars with true Li measurements (filled blue dots and open orange circles in Fig. 13), their $A_{\mathrm{Li}}$ show a significant overall correlation with $[\mathrm{Ba} / \mathrm{Fe}]$ and $[\mathrm{Y} / \mathrm{Fe}]$ (at a $99 \%$ confidence level). Figs. 14 and 15 display the $A_{\mathrm{Li}}-[\mathrm{Ba} / \mathrm{Fe}]$ and $A_{\mathrm{Li}}-$ $[\mathrm{Y} / \mathrm{Fe}]$ correlation, respectively, in different $[\mathrm{Fe} / \mathrm{H}]$ intervals. The anticorrelation is especially significant in the range $-0.74 \lesssim$ $[\mathrm{Fe} / \mathrm{H}] \lesssim 0.43$ for $A_{\mathrm{Li}}-[\mathrm{Ba} / \mathrm{Fe}]$ and $-0.48 \lesssim[\mathrm{Fe} / \mathrm{H}] \lesssim 0.56$ for $A_{\mathrm{Li}}-[\mathrm{Y} / \mathrm{Fe}]$. The Li-s(-process elements) correlation is easily understood since both of them are produced mostly by long-lived stellar sources (see e.g. Romano et al. 1999, 2001; Bisterzo et al. 2017, and references therein).

The main source for $s$-process elements appears to be lowmass AGB stars (Kippenhahn et al. 2012). Given the small 


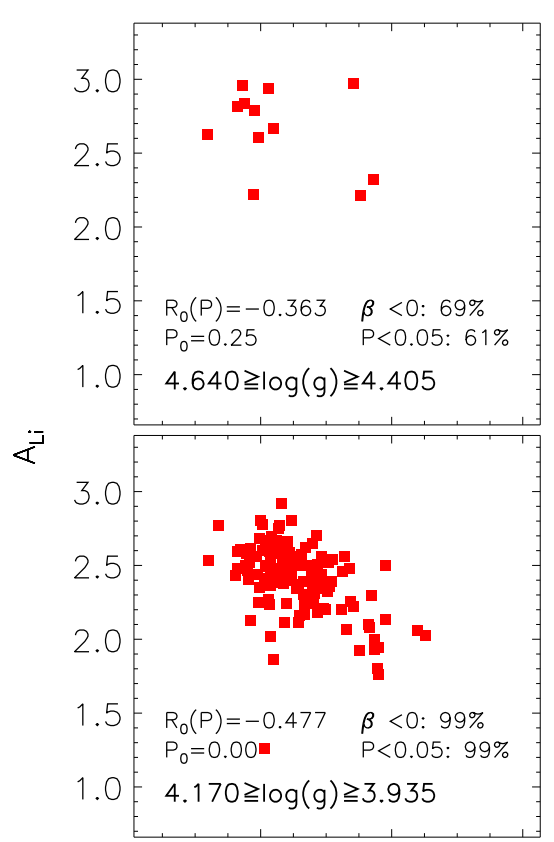

$0.0 \quad 0.3 \quad 0.6$
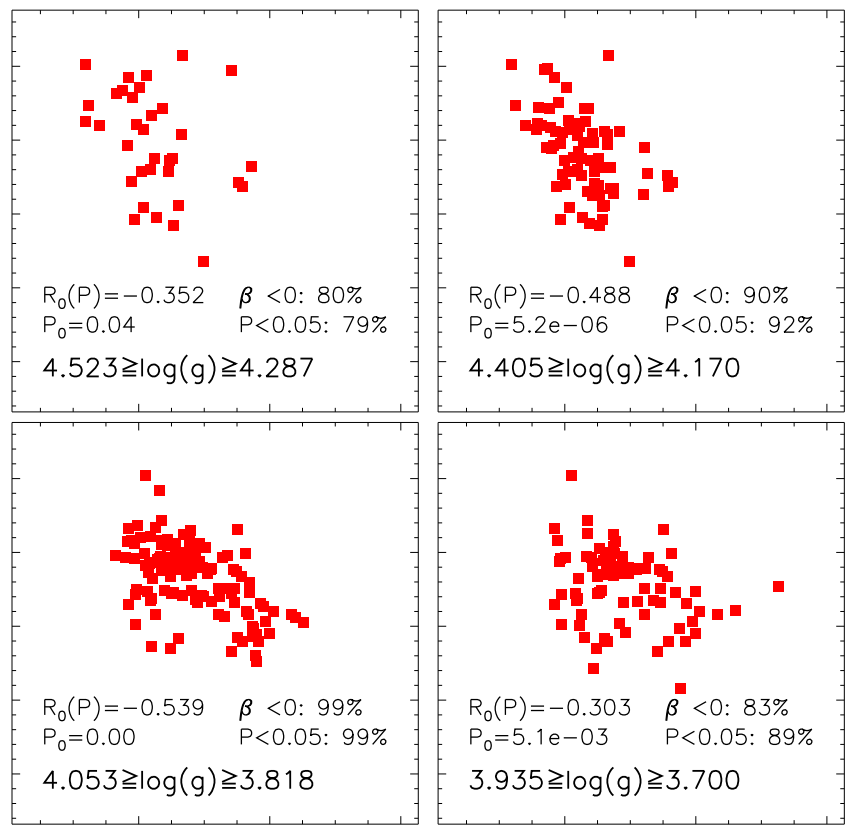

$\begin{array}{lll}0.0 & 0.3 & 0.6 \\ & {[\alpha / \mathrm{Fe}]}\end{array}$

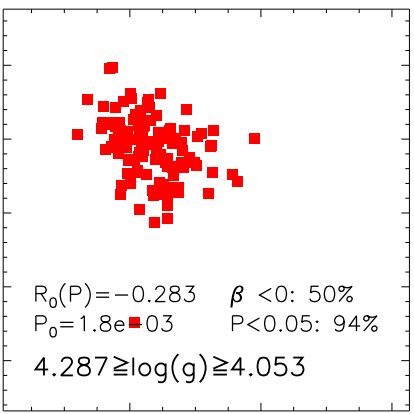

$0.0 \quad 0.3 \quad 0.6$

Fig. 11. NLTE $\mathrm{Li}$ abundance as a function of $[\alpha / \mathrm{Fe}]$ in each $\log (g)$ bin for our sample stars with $\operatorname{Li}$ measurements. The range of $\log (g)$ is specified in each plot, with bin size of $0.235 \mathrm{dex}$. We label the mean correlation coefficient $\left(R_{0}(P)\right)$ between $A_{\mathrm{Li}}$ and $[\alpha / \mathrm{Fe}]$, the mean correlation significance level $\left(P_{0}\right)$, the possibility of $\mathrm{Li}-[\alpha / \mathrm{Fe}]$ anticorrelation $(\beta<0)$ if consider the abundance uncertainties, and the possibility of $P<0.05$ for the anticorrelation.
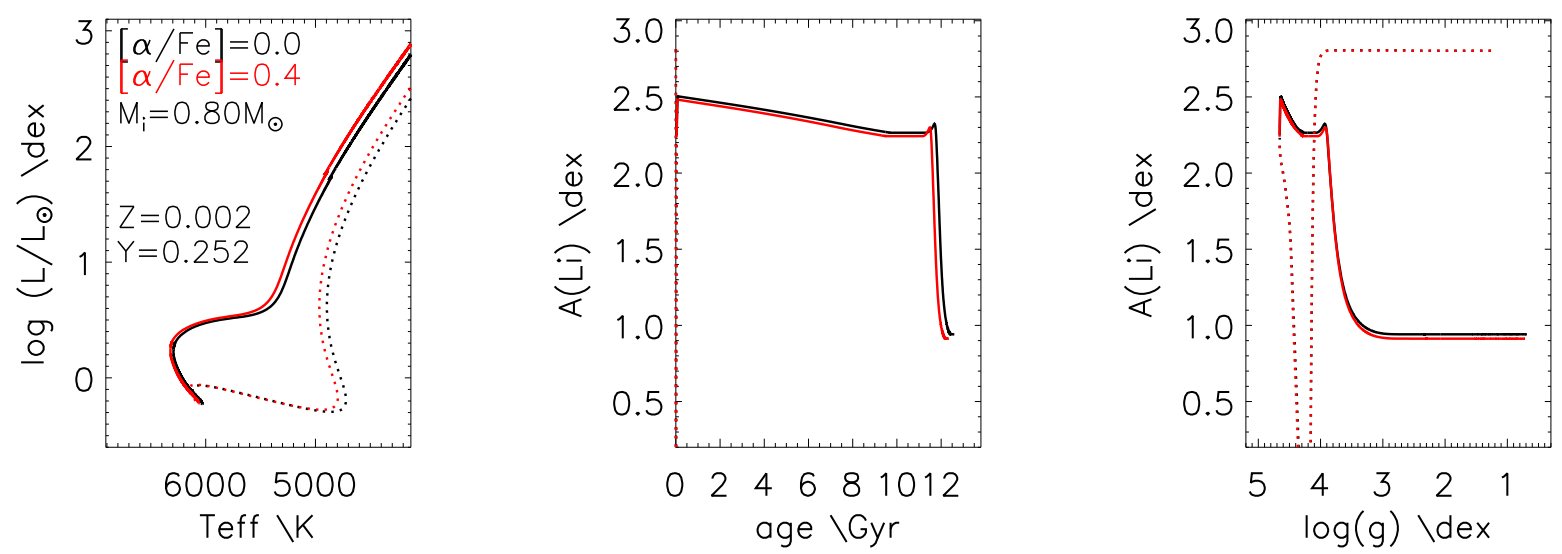

Fig. 12. Li evolution from the stellar evolution model PARSEC. Two stars with the same metallicity and He content $(Z=0.002, Y=0.252)$, the same initial Li abundance $\left(A_{\mathrm{Li}}=2.8 \mathrm{dex}\right)$, the stellar mass $\left(M_{i}=0.80 M_{\odot}\right)$, but different $\alpha$-enhancements are compared. The red curves indicate the star with $[\alpha / \mathrm{Fe}]=0.4$ dex while the black ones are for the star with $[\alpha / \mathrm{Fe}]=0$ (solar-scaled). The dotted lines in both colours represent the evolution before the zero-age main sequence (ZAMS), and the solid lines indicate the evolution after. The panels from left to right are HR diagram, $A_{\mathrm{Li}}$ evolution with stellar age, and $A_{\mathrm{Li}}$ as a function of $\log (g)$.

$[\mathrm{Ba} / \mathrm{Fe}]$ enhancement in most of the stars with lower Li which have higher $[\alpha / \mathrm{Fe}]$ and probably belong to the thick disc, we conclude that AGB stars may mainly contribute to the chemical enrichment in the Galactic thin disc (with lower $[\alpha / \mathrm{Fe}]$ ). Similar results have also been reported by Mashonkina \& Gehren (2001); Delgado Mena et al. (2017) and agree with existing theoretical scenarios of relatively fast/slow thick/thin disc formation (see e.g. Micali et al. 2013).

\section{Discussion}

We show in Sect. 3 that the Galactic thin disc stars have a stronger and higher overall level of Li enrichment than thick disc stars. This result gives us new insight into other observational phenomena, such as the "Li-rich giant problem" (see the Introduction) which should be studied separately in different environments for two reasons. Firstly, the definition of "Li-rich giant" will be affected since the thick and thin disc stars have different initial abundances of $\mathrm{Li}$; secondly, and more importantly, giant stars in the environment with stronger Li enrichment are more likely to be polluted by external sources (i.e. contamination by the ejecta of nearby novae, Martin et al. 1994). Aguilera-Gómez et al. (2016) calculate red giant models with substellar object engulfment and conclude that a Li enrichment up to $A(\mathrm{Li}) \sim 2.2$ dex can be explained by engulfing a 15 Jupiter mass $\left(M_{\mathrm{J}}\right)$ substellar object. If we make a simple calculation, a 

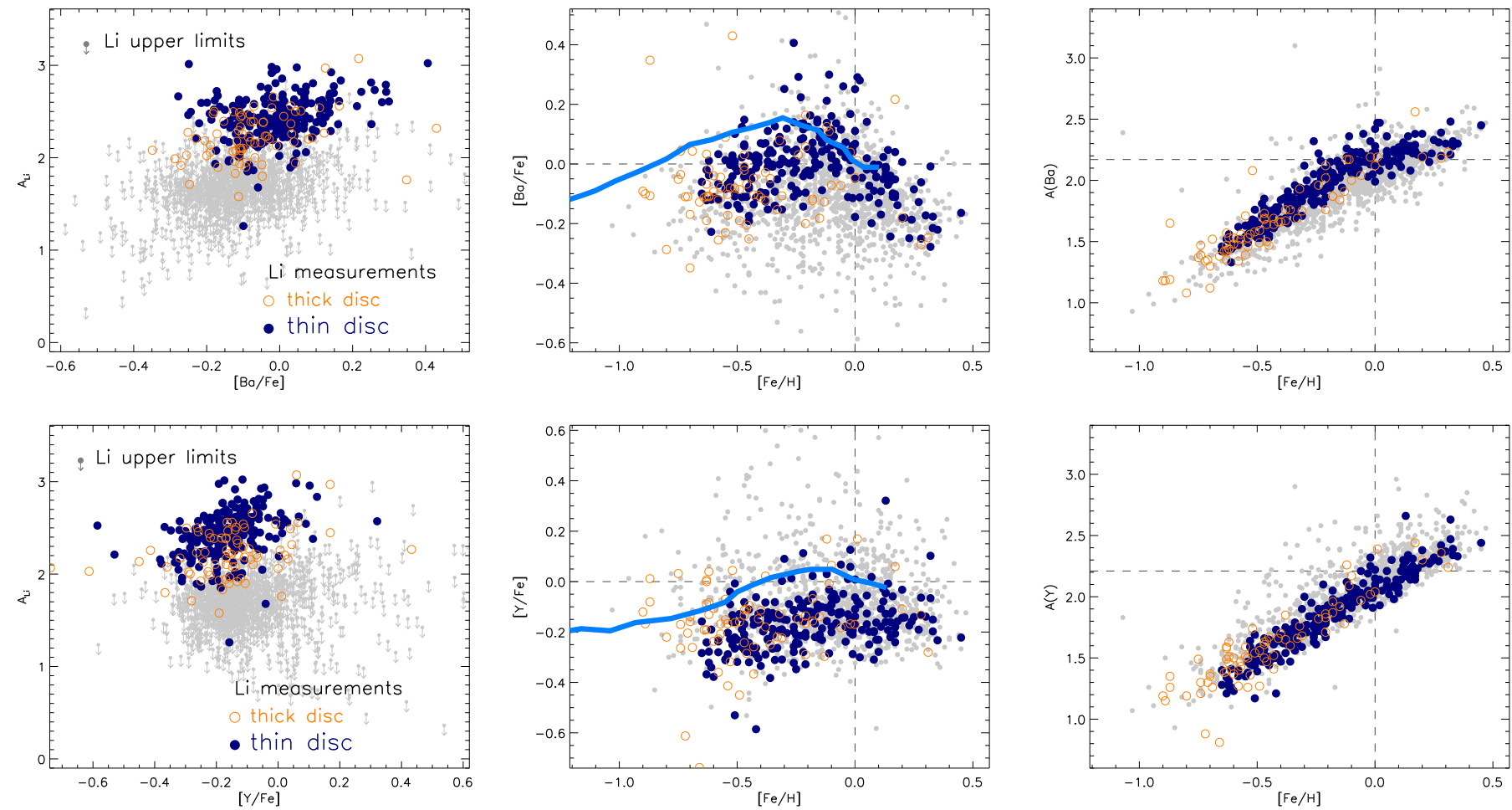

Fig. 13. NLTE Li abundance vs. $[\mathrm{Ba} / \mathrm{Fe}]$ (upper left panel), $[\mathrm{Ba} / \mathrm{Fe}]$ against $[\mathrm{Fe} / \mathrm{H}]$ (upper middle panel), and absolute barium abundance $\mathrm{A}(\mathrm{Ba})$ as a function of $[\mathrm{Fe} / \mathrm{H}]$ (upper right panel). The three lower panels are the same for yttrium. The $[\mathrm{Ba} / \mathrm{Fe}]$ and $[\mathrm{Y} / \mathrm{Fe}] \mathrm{vs}$. $[\mathrm{Fe} / \mathrm{H}]$ trends predicted by Bisterzo et al. (2017) for the Galactic thin disc are overlaid with light blue solid lines. Dashed lines indicate the solar values. Stars with Li measurements are separated as thick (filled blue dots) and thin (open orange circles) disc stars, as the same as in the middle panel of Fig. 6. The grey dots represent sample stars with Li upper limits.

$15 M_{\mathrm{J}}$ substellar object with the initial solar metal mixture contains $M_{\mathrm{Li}} \sim 2 \times 10^{-10} M_{\odot}$, and a single nova outburst can eject $M_{\mathrm{Li}}=0.3 \sim 4.8 \times 10^{-10} M_{\odot}$ (nova V1369 Cen, see Izzo et al. 2015 ) or $M_{\mathrm{Li}} \sim 7 \times 10^{-9} M_{\odot}$ (nova V5668 Sgr, see Molaro et al. 2016). A contamination containing $1 / 30 \sim 7$ single nova ejecta provides the same amount of $\mathrm{Li}$ as a $15 M_{\mathrm{J}}$ substellar object offers. Furthermore, taking into account that $i$. the nova ejecta do not increase the stellar angular momentum that introduces mixing and Li burning, and that $i$. usually a white dwarf can generate multiple novae over time, Li enrichment introduced by novae ejecta contamination is more significant than the substellar objects engulfment. So we expect different frequencies of Li-rich giant stars in different environments.

The upper envelope of the $A_{\mathrm{Li}}$ measurements traced by our sample stars clearly shows that the Galactic Li abundance increases as $[\mathrm{Fe} / \mathrm{H}]$ approaches the solar value, and then declines for super-solar metallicity objects (see Fig. 5, right panel, and Fig. 7, upper panel). A similar trend is seen also in Ramírez et al. (2012); Delgado Mena et al. (2015); Guiglion et al. (2016). Apparently, these findings contrast with classic Galactic chemical evolution model predictions of a monotonic increase of $A_{\mathrm{Li}}$ with metallicity in the Galactic disc (see Romano et al. 1999, 2001; Travaglio et al. 2001; Prantzos 2012). Though metal-rich stars have a deeper surface convective zone compared to the metalpoor ones with the same stellar mass, it is not likely that the $\mathrm{Li}$ decline is due to a stronger Li destruction during the main sequence in the super metal-rich stars because the mean $T_{\text {eff }}$ of these stars are comparable to or higher than the solar metallicity ones (see the lower panel of Fig. 7). From a stellar physics point of view, if the main sequence destruction is not responsible for the $\mathrm{Li}$ decline as explained above, the answer may lie in two fields: $i$. special conditions of mixing and diffusion; and $i$. depletion during the pre-main sequence. For the former, Xiong \& Deng (2009) present a non-local convection stellar model that considers a strong overshooting (the bottom boundary is set at a depth deeper than $5 \times 10^{6} \mathrm{~K}$, which can burn $\mathrm{Li}$ efficiently) and pure gravitational settling of microdiffusion; they predict that $A(\mathrm{Li})$ of warm stars $\left(T_{\text {eff }}>6000 \mathrm{~K}\right)$ decreases with increasing $T_{\text {eff }}$, so stars with high $T_{\text {eff }}$ can also have low $A(\mathrm{Li})$. For the latter, Fu et al. (2015) discuss that $\mathrm{Li}$ in pre-main sequence stars is first depleted by convection then restored by the residual mass accretion, since the very metal-rich disk has high opacity and is easily evaporated; it is possible that the accretion is terminated early and the $\mathrm{Li}$ is not fully restored. In the following, we discuss some possible explanations from the perspective of the Galactic chemical evolution for the observed Li decrease in super-solar metallicity stars, in the framework of the model by Romano et al. (1999, 2001).

In particular, from a close inspection of Figs. 3 and 4 of Romano et al. (2001), we note that the Li abundance increases with metallicity until $[\mathrm{Fe} / \mathrm{H}] \sim 0$, but then decreases for $[\mathrm{Fe} / \mathrm{H}]>$ 0 , in agreement with the trend suggested by our observations. A similar prediction trend is also seen in Fig. 5 of Abia et al. (1998) with a different choice of yields. The predicted behaviour in Romano et al. (2001, with contributions from both AGB stars and core-collapse $\mathrm{SNe}$ ) is due to the presence of a threshold in the gas density in their model, below which the star formation stops (see also Chiappini et al. 2001, and reference therein). During the last $\sim 5$ Gyr of evolution, due to the presence of such a threshold, the star formation in the solar neighbourhood has several short periods of activity intercut with star formation gaps. As a consequence, the restitution rate of Li from AGB stars and core-collapse $\mathrm{SNe}$, which tracks rather closely the star formation rate owing to the relatively short lifetimes of the stellar 


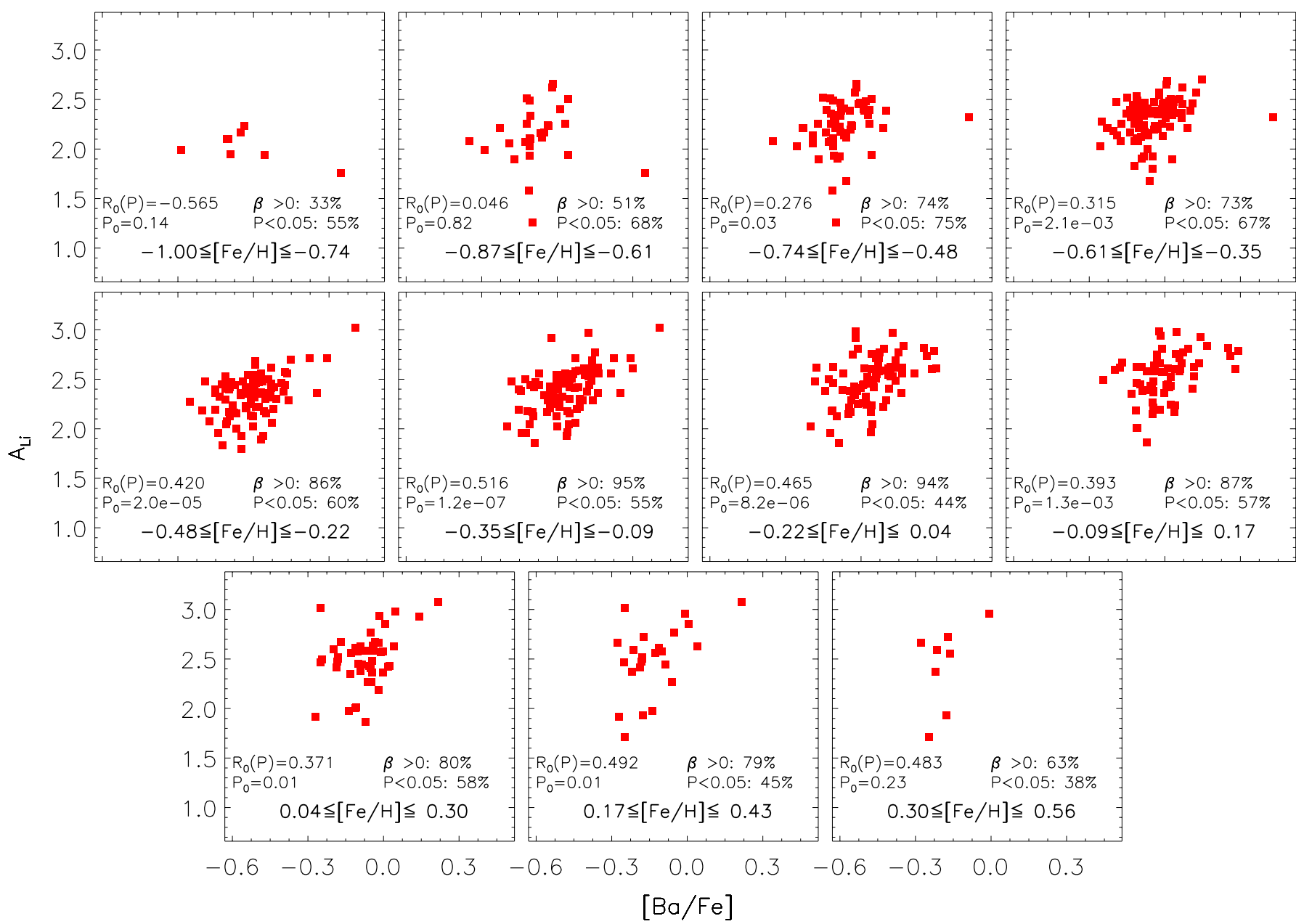

Fig. 14. NLTE Li abundance as a function of $[\mathrm{Ba} / \mathrm{Fe}]$ in each $[\mathrm{Fe} / \mathrm{H}]$ bin for our sample stars with $\mathrm{Li}$ measurements. The range of $[\mathrm{Fe} / \mathrm{H}]$ is specified in each plot. The $[\mathrm{Fe} / \mathrm{H}]$ bin size is 0.26 dex. In each bin we label the mean correlation coefficient $\left(R_{0}(P)\right)$ between $A_{\mathrm{Li}}$ and $[\mathrm{Ba} / \mathrm{Fe}]$, the mean correlation significance level $\left(P_{0}\right)$, the possibility of $A_{\mathrm{Li}}-[\mathrm{Ba} / \mathrm{Fe}]$ correlation $(\beta>0)$ if considering the abundance uncertainties, and the possibility of $P<0.05$ for the anticorrelation.

progenitors, is considerably reduced, and the stellar Li astration eventually overcomes the Li production. This star formation gap explanation may hold as well for pure $s$-process elements synthesised in low-mass stars, and explains why their abundance relative to iron decreases in super-solar metallicity stars (Fig. 13, the two middle panels; see also Bensby et al. 2005; Bisterzo et al. 2017; Delgado Mena et al. 2017). Furthermore, the absolute abundances of barium show a kind of plateau at super-solar metallicity (see the upper-left panel of Fig. 13), which strongly indicates that the production of the $s$-process elements was minor, or even stopped for some time. However, though the NLTE correction has little influence on Ba abundance (Mishenina et al. 2015), stellar activities might affect the A(Ba) trend. Reddy \& Lambert (2017) present a strong correlation between the $[\mathrm{Ba} \mathrm{II} / \mathrm{Fe}]$ values and the chromospheric activity for solar-twin stars. These authors demonstrate that the Ba II abundances of these stars are overestimated because of the adoption of an overly low value of microturbulence in the spectrum synthesis. Unfortunately, we are not able to confirm this effect in our sample stars because the stellar activity information is not available. It should also be stressed that the yields of super-solar metallicity stars are not widely investigated in the literature; the yields of $s$-process elements are not very clear. For example, Cristallo et al. (2015a) demonstrate that the yields of the second $s$-process peak (including Ba) mainly contributes to $-0.7<[\mathrm{Fe} / \mathrm{H}]<-0.3$ and decreases its contribution at higher metallicities, while Karakas \& Lugaro (2016) show that the yields of $\mathrm{Ba}$ increase from $Z=0.007$ to $Z=0.03$. Further complicating matters, one must consider also the contribution of low-mass stars that die when the metallicity of the ISM is super solar but were born at earlier stages from a metal-poor ISM. Such work requires thorough calculations in the Galactic chemical models.

Nevertheless, precise measurements of Li and s-process element abundances in stars will be extremely useful to constrain the AGB yields, as well as the Galactic chemical evolution models.

As recently confirmed by the direct detection of $\mathrm{Li}$ in the spectra of nova V1369 Cen by Izzo et al. (2015), given the current estimates of the Galactic nova rate, novae might contribute most of the $\mathrm{Li}$ in the Galaxy. Particularly slow novae, with a rate of around 17 events $\mathrm{yr}^{-1}$, would eject Li into the ISM at amounts significantly larger than their fast counterparts (Izzo et al. 2015). The nova rate is highly sensitive to the assumed fraction and mass distribution of the binary stellar progenitors. In particular, Gao et al. (2014, 2017), Yuan et al. (2015) report that the fraction of close binaries (which lead to nova outbursts) decreases with increasing $[\mathrm{Fe} / \mathrm{H}]$. The super-Chandrasekhar SNe Ia, an end result of binary stars, also strongly prefer metal-poor environments (Khan et al. 2011). These observations imply that the 


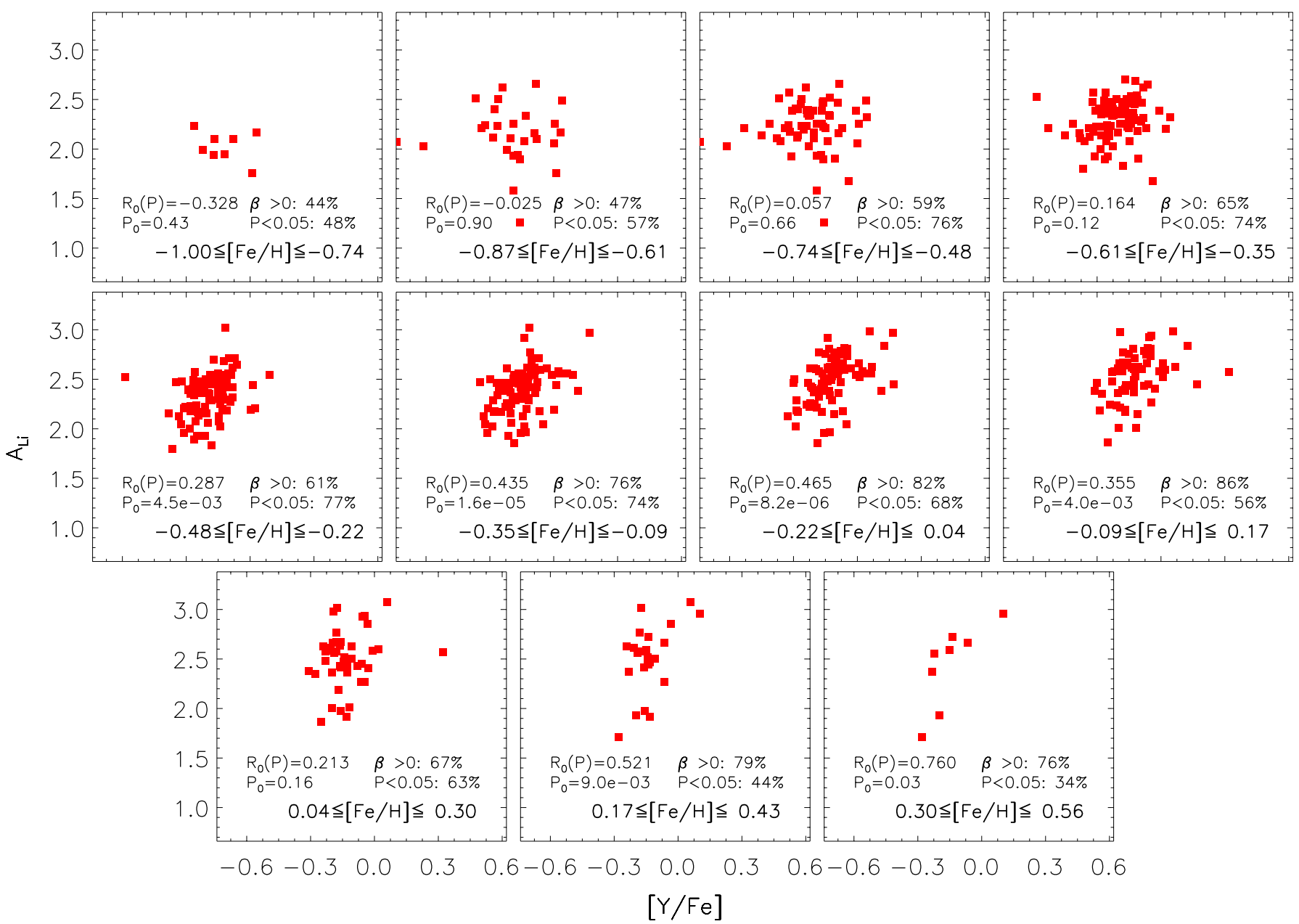

Fig. 15. NLTE Li abundance as a function of $[\mathrm{Y} / \mathrm{Fe}]$ in each $[\mathrm{Fe} / \mathrm{H}]$ bin for our sample stars with Li measurements. The range of $[\mathrm{Fe} / \mathrm{H}]$ is specified in each plot. The $[\mathrm{Fe} / \mathrm{H}]$ bin size is 0.26 dex. In each bin we label the mean correlation coefficient $\left(R_{0}(P)\right)$ between $A_{\mathrm{Li}}$ and $[\mathrm{Y} / \mathrm{Fe}]$, the mean correlation significance level $\left(P_{0}\right)$, the possibility of $A_{\mathrm{Li}}-[\mathrm{Y} / \mathrm{Fe}]$ correlation $(\beta>0)$ if consider the abundance uncertainties, and the possibility of $P<0.05$ for the anticorrelation.

occurrence of nova systems may be lower at higher metallicities, which would lower the total $\mathrm{Li}$ production from such objects in high-metallicity environments. Such a metal-dependence of the nova system formation rate has not been taken into account in Galactic chemical evolution models up to now (Romano et al. 2001; Travaglio et al. 2001). Here we suggest that it could be (jointly) responsible for the observed decreasing trend of $A_{\mathrm{Li}}$ for $[\mathrm{Fe} / \mathrm{H}]>0$, a hypothesis that needs to be tested by means of detailed chemical evolution models.

\section{Summary}

We investigate the Li enrichment histories in the Galactic discs using GES iDR4 data. Li abundance, $[\alpha / \mathrm{Fe}],[\mathrm{Ba} / \mathrm{Fe}]$, and $[\mathrm{Y} / \mathrm{Fe}]$ for 1399 well-measured $([\mathrm{Fe} / \mathrm{H}]$ error $<0.13 \mathrm{dex})$ main sequence $(\log (g) \leq 3.7 \mathrm{dex})$ field stars with UVES spectra are studied. We divide the sample stars into two categories: $\mathrm{Li}$ measurements and $\mathrm{Li}$ upper limits. NLTE corrections are applied to the $\mathrm{Li}$ line at $6708 \AA$. Four $\alpha$ elements, $\mathrm{Mg}, \mathrm{Si}$, $\mathrm{Ca}$, and $\mathrm{Ti}$, are considered in the MCMC calculations to derive the total $[\alpha / \mathrm{Fe}]$ and the corresponding $1 \sigma$ uncertainty. We find a Li- $[\alpha / \mathrm{Fe}]$ anticorrelation independent of $[\mathrm{Fe} / \mathrm{H}], T_{\text {eff }}$, and $\log (g)$ in our sample stars with actual Li measurements. After checking the behaviour of surface $\mathrm{Li}$ in stellar evolution models we conclude that different $\alpha$ enhancements do not lead to measurable $A_{\mathrm{Li}}$ differences, thus the $\mathrm{Li}-[\alpha / \mathrm{Fe}]$ anticorrelation echos different levels of $\mathrm{Li}$ enrichments in these stars. A Li-s(-process elements) correlation, which is connected to the nucleosynthesis in their common production site (AGB stars) is also seen.

We perform a tentative division based on $[\alpha / \mathrm{Fe}]$ and $[\mathrm{Fe} / \mathrm{H}]$ in order to separate our sample into thick disc stars and thin disc ones. By comparing, in each $[\mathrm{Fe} / \mathrm{H}]$ bin, the error-weighted mean, $A_{\mathrm{Li}}$ and $T_{\mathrm{eff}}$ values of stars with the highest $\mathrm{Li}$ abundance, as well as the fractions of Li-enriched stars, we conclude that the thin disc stars experience a stronger Li enrichment throughout their evolution, compared to the thick disc stars.

The Li decline in super-solar metallicity, which has already been reported by Delgado Mena et al. (2015) and Guiglion et al. (2016), is confirmed by the GES analysis. We discuss the possible explanations of this scenario. It may be a joint consequence of AGB yield evolution and the low binary fraction at a high metallicity.

Acknowledgements. This work is based on data products from observations made with ESO Telescopes at the La Silla Paranal Observatory under programme ID 188.B-3002 and 193.B-0936. These data products have been processed by the Cambridge Astronomy Survey Unit (CASU) at the Institute of Astronomy, University of Cambridge, and by the FLAMES/UVES reduction team at INAF/Osservatorio Astrofisico di Arcetri. Data used her were obtained from the Gaia-ESO Survey Data Archive, prepared and hosted by the Wide Field 
Astronomy Unit, Institute for Astronomy, University of Edinburgh, which is funded by the UK Science and Technology Facilities Council. This work was partly supported by the European Union FP7 programme through ERC grant number 320360 and by the Leverhulme Trust through grant RPG-2012-541. We acknowledge the support from INAF and Ministero dell' Istruzione, dell' Università' e della Ricerca (MIUR) in the form of the grant "Premiale VLT 2012". The results presented here benefit from discussions held during the Gaia-ESO workshops and conferences supported by the ESF (European Science Foundation) through the GREAT Research Network Programme. This research has made use of the TOPCAT catalogue handling and plotting tool (Taylor 2005, 2017); of the Simbad database and the VizieR catalogue access tool, CDS, Strasbourg, France (Ochsenbein et al. 2000); and of NASA's Astrophysics Data System. X.F acknowledges helpful discussions with Nikos Prantzos and Paolo Molaro, and thanks Zhiyu Zhang for the help on MCMC calculations. E.D.M. and S.G.S. acknowledge the support from Fundaçäo para a Ciência e a Tecnologia (FCT) through national funds and from FEDER through COMPETE2020 by the following grants: UID/FIS/04434/2013 \& POCI-01-0145-FEDER007672, PTDC/FIS-AST/1526/2014 \& POCI-01-0145-FEDER-016886, and PTDC/FIS-AST/7073/2014 \& POCI-01-0145-FEDER-016880. E.D.M. and S.G.S. also acknowledge the support from FCT through Investigador FCT contracts IF/00849/2015/CP1273/CT003 and IF/00028/2014/CP1215/CT0002. C.A acknowledges to the Spanish grant AYA2015-63588-P within the European Founds for Regional Development (FEDER). A.K. and T.B. acknowledge the project grant "The New Milky Way" from the Knut and Alice Wallenberg Foundation. R.S. acknowledges support from the Polish Ministry of Science and Higher Education.

\section{References}

Abia, C., Isern, J., \& Canal, R. 1993, A\&A, 275, 96

Abia, C., Isern, J., \& Lisenfeld, U. 1998, MNRAS, 299, 1007

Adibekyan, V. Z., Sousa, S. G., Santos, N. C., et al. 2012, A\&A, 545, A32

Aguilera-Gómez, C., Chanamé, J., Pinsonneault, M. H., \& Carlberg, J. K. 2016, ApJ, 829, 127

Aoki, W., Ito, H., \& Tajitsu, A. 2012, ApJ, 751, L6

Asplund, M., Lambert, D. L., Nissen, P. E., Primas, F., \& Smith, V. V. 2006, ApJ, 644, 229

Balachandran, S. C., Fekel, F. C., Henry, G. W., \& Uitenbroek, H. 2000, ApJ, 542,978

Bensby, T., Feltzing, S., Lundström, I., \& Ilyin, I. 2005, A\&A, 433, 185

Bensby, T., Feltzing, S., \& Oey, M. S. 2014, A\&A, 562, A71

Bisterzo, S., Travaglio, C., Wiescher, M., Käppeler, F., \& Gallino, R. 2017, ApJ, 835,1

Bonifacio, P., \& Molaro, P. 1997, MNRAS, 285, 847

Bonifacio, P., Molaro, P., Sivarani, T., et al. 2007, A\&A, 462, 851

Bonifacio, P., Caffau, E., Spite, M., et al. 2015, A\&A, 579, A28

Bonsack, W. K., \& Greenstein, J. L. 1960, ApJ, 131, 83

Bressan, A., Marigo, P., Girardi, L., et al. 2012, MNRAS, 427, 127

Cameron, A. G. W., \& Fowler, W. A. 1971, ApJ, 164, 111

Casey, A. R., Ruchti, G., Masseron, T., et al. 2016, MNRAS, 461, 3336

Cescutti, G., François, P., Matteucci, F., Cayrel, R., \& Spite, M. 2006, A\&A, 448,557

Charbonnel, C., \& Zahn, J. 2007, A\&A, 18, 15

Chen, Y. Q., Nissen, P. E., Benoni, T., \& Zhao, G. 2001, A\&A, 371, 943

Chiappini, C., Matteucci, F., \& Romano, D. 2001, ApJ, 554, 1044

Coc, A., Uzan, J.-P., \& Vangioni, E. 2014, J. Cosmol. Astropart. Phys., 10, 050

Cristallo, S., Abia, C., Straniero, O., \& Piersanti, L. 2015a, ApJ, 801, 53

Cristallo, S., Straniero, O., Piersanti, L., \& Gobrecht, D. 2015b, ApJS, 219, 40

Dekker, H., D’Odorico, S., Kaufer, A., Delabre, B., \& Kotzlowski, H. 2000, in Optical and IR Telescope Instrumentation and Detectors, eds. M. Iye, \& A. F. Moorwood, Proc. SPIE, 4008, 534

Delgado Mena, E., Bertrán de Lis, S., Adibekyan, V. Z., et al. 2015, A\&A, 576, A69

Delgado Mena, E., Tsantaki, M., Adibekyan, V. Z., et al. 2017, A\&A, 606, A94

Domínguez, I., Abia, C., Straniero, O., Cristallo, S., \& Pavlenko, Y. V. 2004 A\&A, 422, 1045

Domogatskii, G. V., Eramzhian, R. A., \& Nadezhin, D. K. 1978, Ap\&SS, 58, 273

D’Orazi, V., Gratton, R. G., Angelou, G. C., et al. 2015, ApJ, 801, L32

Dotter, A., Conroy, C., Cargile, P., \& Asplund, M. 2017, ApJ, 840, 99

Foreman-Mackey, D., Hogg, D. W., Lang, D., \& Goodman, J. 2013, PASP, 125 , 306

Fu, X. 2016, Ph.D. Thesis, SISSA - International School for Advanced Studies, Via Bonomea 265, 34136 Trieste, Italy

Fu, X., Bressan, A., Molaro, P., \& Marigo, P. 2015, MNRAS, 452, 3256

Fu, X., Bressan, A., Marigo, P., et al. 2017, submitted

Fuhrmann, K. 1998, A\&A, 338, 161
Gaia Collaboration, Brown, A. G. A., Vallenari, A., et al. 2016, A\&A, 595, A2 Gao, S., Liu, C., Zhang, X., et al. 2014, ApJ, 788, L37

Gao, S., Zhao, H., Yang, H., \& Gao, R. 2017, MNRAS, 469, L68 Gilmore, G., Randich, S., Asplund, M., et al. 2012, The Messenger, 147, 25 González Hernández, J. I., Bonifacio, P., Ludwig, H.-G., et al. 2008, A\&A, 480, 233

Gratton, R. G., Carretta, E., Matteucci, F., \& Sneden, C. 2000, A\&A, 358, 671 Grevesse, N., Asplund, M., \& Sauval, A. J. 2007, Space Sci. Rev., 130, 105 Guiglion, G., de Laverny, P., Recio-Blanco, A., et al. 2016, A\&A, 595, A18 Gustafsson, B., Edvardsson, B., Eriksson, K., et al. 2008, A\&A, 486, 951 Hansen, T., Hansen, C. J., Christlieb, N., et al. 2014, ApJ, 787, 162 Haywood, M., Di Matteo, P., Lehnert, M. D., Katz, D., \& Gómez, A. 2013, A\&A, 560, A109

Heiter, U., Lind, K., Asplund, M., et al. 2015, Phys. Scr., 90, 054010 Herbig, G. H. 1965, ApJ, 141, 588

Hill, V., \& Pasquini, L. 1999, A\&A, 348, L21

Iben, Icko, Jr. 1967a, ApJ, 147, 650

Iben, Icko, Jr. 1967b, ApJ, 147, 624

Israelian, G., Bertran de Lis, S., Delgado Mena, E., \& Adibekyan, V. Z. 2014, Mem. Soc. Astron. Italiana, 85, 265

Izzo, L., Della Valle, M., Mason, E., et al. 2015, ApJ, 808, L14

Karakas, A. I., \& Lugaro, M. 2016, ApJ, 825, 26

Kelly, B. C. 2007, ApJ, 665, 1489

Khan, R., Stanek, K. Z., Stoll, R., \& Prieto, J. L. 2011, ApJ, 737, L24

Kippenhahn, R., Weigert, A., \& Weiss, A. 2012, Stellar Structure and Evolution (Berlin Heidelberg: Springer-Verlag)

Kirby, E. N., Fu, X., Guhathakurta, P., \& Deng, L. 2012, ApJ, 752, L16

Kirby, E. N., Guhathakurta, P., Zhang, A. J., et al. 2016, ApJ, 819, 135

Korn, a. J., Grundahl, F., Richard, O., et al. 2007, ApJ, 671, 402

Kraft, R. P., Peterson, R. C., Guhathakurta, P., et al. 1999, ApJ, 518, L53

Kumar, Y. B., \& Reddy, B. E. 2009, ApJ, 703, L46

Lambert, D. L., \& Reddy, B. E. 2004, MNRAS, 349, 757

Lemoine, M., Vangioni-Flam, E., \& Cassé, M. 1998, ApJ, 499, 735

Lind, K., Asplund, M., \& Barklem, P. S. 2009, A\&A, 503, 541

Lodders, K., Palme, H., \& Gail, H.-P. 2009, Landolt Börnstein [arXiv: 0901.1149]

Maiorca, E., Magrini, L., Busso, M., et al. 2012, ApJ, 747, 53

Martin, E. L., Rebolo, R., Casares, J., \& Charles, P. A. 1994, ApJ, 435, 791

Mashonkina, L., \& Gehren, T. 2001, A\&A, 376, 232

Matteucci, F. 2012, Chemical Evolution of Galaxies (Berlin Heidelberg: Springer-Verlag)

Matteucci, F., \& Greggio, L. 1986, A\&A, 154, 279

Melendez, J., Casagrande, L., Ramirez, I., Asplund, M., \& Schuster, W. 2010, A\&A, 515, L3

Meneguzzi, M., Audouze, J., \& Reeves, H. 1971, A\&A, 15, 337

Micali, A., Matteucci, F., \& Romano, D. 2013, MNRAS, 436, 1648

Michalik, D., Lindegren, L., \& Hobbs, D. 2015, A\&A, 574, A115

Mikolaitis, S., Hill, V., Recio-Blanco, A., et al. 2014, A\&A, 572, A33

Mishenina, T., Pignatari, M., Carraro, G., et al. 2015, MNRAS, 446, 3651

Molaro, P., Izzo, L., Mason, E., Bonifacio, P., \& Della Valle, M. 2016, MNRAS, 463, L117

Monaco, L., Villanova, S., Moni Bidin, C., et al. 2011, A\&A, 529, A90

Nordlander, T., Korn, A. J., Richard, O., \& Lind, K. 2012, ApJ, 753, 48

Ochsenbein, F., Bauer, P., \& Marcout, J. 2000, A\&AS, 143, 23

Pagel, B. E. J., \& Tautvaisiene, G. 1997, MNRAS, 288, 108

Pancino, E., Lardo, C., Altavilla, G., et al. 2017, A\&A, 598, A5

Pasquini, L., Avila, G., Allaert, E., et al. 2000, in Optical and IR Telescope Instrumentation and Detectors, eds. M. Iye, \& A. F. Moorwood, Proc. SPIE, 4008, 129

Pilachowski, C. 1986, ApJ, 300, 289

Prantzos, N. 2012, A\&A, 542, A67

Ramírez, I., Fish, J. R., Lambert, D. L., \& Allende Prieto, C. 2012, ApJ, 756, 46

Randich, S., Gilmore, G., \& Gaia-ESO Consortium. 2013, The Messenger, 154, 47

Recio-Blanco, A., de Laverny, P., Kordopatis, G., et al. 2014, A\&A, 567, A5

Reddy, A. B. S., \& Lambert, D. L. 2017, ApJ, 845, 151

Reddy, B. E., Tomkin, J., Lambert, D. L., \& Prieto, C. A. 2003, MNRAS, 340, 304

Reeves, H., Fowler, W. A., \& Hoyle, F. 1970, Nature, 226, 727

Richard, O., Michaud, G., Richer, J., et al. 2002, ApJ, 568, 979

Rojas-Arriagada, A., Recio-Blanco, A., de Laverny, P., et al. 2017, A\&A, 601, A140

Romano, D., Matteucci, F., Molaro, P., \& Bonifacio, P. 1999, A\&A, 352, 117 Romano, D., Matteucci, F., Ventura, P., \& D’Antona, F. 2001, A\&A, 374, 646 Ruffoni, M. P., Den Hartog, E. A., Lawler, J. E., et al. 2014, MNRAS, 441, 3127 Sacco, G. G., Morbidelli, L., Franciosini, E., et al. 2014, A\&A, 565, A113

Sackmann, I.-J., \& Boothroyd, A. I. 1992, ApJ, 392, L71

Sackmann, I.-J., \& Boothroyd, A. I. 1999, ApJ, 510, 217 
Sbordone, L., Bonifacio, P., Caffau, E., et al. 2010, A\&A, 522, A26 Schlegel, D. J., Finkbeiner, D. P., \& Davis, M. 1998, ApJ, 500, 525 Skrutskie, M. F., Cutri, R. M., Stiening, R., et al. 2006, AJ, 131, 1163 Smiljanic, R., Korn, A. J., Bergemann, M., et al. 2014, A\&A, 570, A122 Spite, F., \& Spite, M. 1982, A\&A, 115, 357

Starrfield, S., Truran, J. W., Sparks, W. M., \& Arnould, M. 1978, ApJ, 222, 600 Stonkute, E., Koposov, S. E., Howes, L. M., et al. 2016, MNRAS, 460, 1131

Tajitsu, A., Sadakane, K., Naito, H., Arai, A., \& Aoki, W. 2015, Nature, 518, 381 Tajitsu, A., Sadakane, K., Naito, H., et al. 2016, ApJ, 818, 191

Taylor, M. 2017, Informatics, 4, 18

Taylor, M. B. 2005, Astronomical Data Analysis Software and Systems XIV, ASP Conf. Ser., 347, 29

Thoul, A. A., Bahcall, J. N., \& Loeb, A. 1994, ApJ, 421, 828

Tinsley, B. M. 1979, ApJ, 229, 1046

Travaglio, C., Galli, D., Gallino, R., et al. 1999, ApJ, 521, 691

Travaglio, C., Randich, S., Galli, D., et al. 2001, ApJ, 559, 909

Travaglio, C., Gallino, R., Arnone, E., et al. 2004, ApJ, 601, 864

Vangioni-Flam, E., Cassé, M., Fields, B. D., \& Olive, K. A. 1996, ApJ, 468, 199

Venn, K. A., Irwin, M., Shetrone, M. D., et al. 2004, AJ, 128, 1177

Wallerstein, G., Herbig, G. H., \& Conti, P. S. 1965, ApJ, 141, 610

Wallerstein, G., \& Sneden, C. 1982, ApJ, 255, 577

Xiong, D. R., \& Deng, L. 2009, MNRAS, 395, 2013

Yuan, H., Liu, X., Xiang, M., et al. 2015, ApJ, 799, 135

${ }^{1}$ Dipartimento di Fisica \& Astronomia, Università degli Studi di Bologna, via Gobetti 93/2, 40129 Bologna, Italy

2 INAF-Osservatorio Astronomico di Bologna, via Gobetti 93/3, 40129 Bologna, Italy

e-mail: xiaoting. fu@oabo.inaf.it

${ }^{3}$ Max-Planck Institut für Astronomie, Königstuhl 17, 69117 Heidelberg, Germany

${ }^{4}$ Instituto de Astrofísica e Cências do Espaço, Universidade do Porto, CAUP, Rua das Estrelas, 4150-762 Porto, Portugal

5 INAF-Osservatorio Astrofisico di Arcetri, Largo E. Fermi 5, 50125 Florence, Italy

${ }^{6}$ SISSA-International School for Advanced Studies, via Bonomea 265, 34136 Trieste, Italy
${ }^{7}$ European Southern Observatory, Alonso de Cordova 3107 Vitacura, Santiago de Chile, Chile

8 University of New South Wales Sydney, Australia 2052

9 Department of Physics and Astronomy, Uppsala University, Box 516, 75120 Uppsala, Sweden

${ }^{10}$ Dpto. Física Teǿrica y del Cosmos, Universidad de Granada, 18071 Granada, Spain

11 Nicolaus Copernicus Astronomical Center, Polish Academy of Sciences, ul. Bartycka 18, 00-716 Warsaw, Poland

12 Institute of Astronomy, University of Cambridge, Madingley Road, Cambridge CB3 0HA, UK

13 Institute of Theoretical Physics and Astronomy, Vilnius University, Saulètekio av. 3, 10257 Vilnius, Lithuania

14 Departamento de Astronomia, Universidad de Concepcion, 3349001 Concepcion, Chile

15 Dipartimento di Fisica e Astronomia, Sezione Astrofisica, Universitá di Catania, via S. Sofia 78, 95123 Catania, Italy

16 Dipartimento di Fisica e Astronomia, Università di Padova, Vicolo Osservatorio 3, 35122 Padova, Italy

17 Lund Observatory, Department of Astronomy and Theoretical Physics, Box 43, 22100 Lund, Sweden

18 INAF-Osservatorio Astronomico di Palermo, Piazza del Parlamento 1, 90134 Palermo, Italy

19 Instituto de Astrofísica de Andalucía-CSIC, Apdo. 3004, 18080 Granada, Spain

20 INAF-Padova Observatory, Vicolo dell'Osservatorio 5, 35122 Padova, Italy

${ }^{21}$ Laboratoire d'astrophysique, Ecole Polytechnique Fédérale de Lausanne (EPFL), Observatoire de Sauverny, 1290 Versoix, Switzerland

22 Departamento de Ciencias Fisicas, Universidad Andres Bello, Fernandez Concha 700, Las Condes, Santiago, Chile

23 Instituto de Física y Astronomiía, Universidad de Valparaiíso, Chile

24 Observatoire de la Côte d'Azur, Laboratoire Lagrange, CNRS UMR 7293, Nice Cedex 04, France

25 Nucleo de Astronomia, Universidad Diego Portales, Ejercito 441, Santiago, Chile 